\title{
Selective Addition to Iridium of Aryl C-H Bonds Ortho to Coordinating Groups. Not Chelation-Assisted
}

\author{
Xiawei Zhang, Mira Kanzelberger, Thomas J. Emge and Alan S. Goldman* \\ Department of Chemistry and Chemical Biology, \\ Rutgers-The State University of New Jersey, Piscataway, New Jersey, 08854
}

\section{Supporting Information}

Page \#

I. Methods, Spectroscopic and Analytical Data

S2

II. Structural Data for 1a

(a) Figure S-1. ORTEP Diagrams of 1a

S5

(b) Table S-1. Crystal data and structure refinement for $\mathbf{1 a}$

S6

(c) Table S-2. Atomic coordinates for 1a

S7

(d) Table S-3. Selected bond lengths and angles for 1a

S8

III. Structural Data for $\mathbf{1 b}$

(a) Figure S-2. ORTEP Diagrams of $\mathbf{1 b}$

S9

(b) Table S-4. Crystal data and structure refinement for $\mathbf{1 b}$

S10

(c) Table S-5. Atomic coordinates for $\mathbf{1 b}$

S11

(d) Table S-6. Selected bond lengths and angles for $\mathbf{1 b}$

S12

IV. Structural Data for $\mathbf{2 a}$

(a) Figure S-3. ORTEP Diagrams of 2a

S13

(b) Table S-7. Crystal data and structure refinement for $\mathbf{2 a}$

$\mathrm{S} 14$

(c) Table S-8. Atomic coordinates for $\mathbf{2 a}$

S15

(d) Table S-9. Selected bond lengths and angles for $\mathbf{2 a}$

S16

V. Structural Data for $\mathbf{2 b}$

(a) Figure S-4. ORTEP Diagrams of $\mathbf{2 b}$

(b) Table S-10. Crystal data and structure refinement for $\mathbf{2 b}$

S18

(c) Table S-11. Atomic coordinates for $\mathbf{2 b}$

S19

(d) Table S-12. Selected bond lengths and angles for $\mathbf{2 b}$ 


\section{Methods, Spectroscopic and Analytical Data}

General Methods. Unless otherwise noted, all reactions, recrystallizations and routine manipulations were performed at ambient temperature in an argon-filled glovebox, or by using standard Schlenk techniques. Kinetic experiments were carried out in J-Young NMR tubes in deuterated solvents and were monitored by ${ }^{1} \mathrm{H}$ and ${ }^{31} \mathrm{P}$ NMR spectroscopy.

Reagent grade solvents were dried according to established methods, then degassed with argon. All NMR solvents were dried, vacuum-transferred, and stored in an argon-filled glove box. (PCP) $\mathrm{IrH}_{2}{ }^{1}$ and 4-d-nitrobenzene ${ }^{2}$ were prepared according to published methods. All other chemicals were used as received from commercial suppliers.

${ }^{1} \mathrm{H}$ and ${ }^{31} \mathrm{P}$ NMR spectra were obtained on a 300-MHz Varian Mercury $300 .{ }^{31} \mathrm{P}$ NMR chemical shifts are referenced to an $85 \% \mathrm{H}_{3} \mathrm{PO}_{4}$ solution. Infrared (IR) spectra were recorded on an ATI Mattson Genesis Series FTIR in pentane solutions.

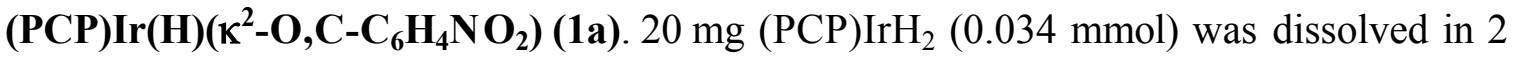
$\mathrm{mL} p$-xylene solution containing $5.0 \mathrm{mg}$ norbornene $(0.053 \mathrm{mmol})$. To the resulting solution was added $6.0 \mu \mathrm{L}$ nitrobenzene $(0.052 \mathrm{mmol})$; after stirring for ca. $1 \mathrm{~min}$, the red solution turned deeper red. Solvent was evacuated and the resulting solid was redissolved in and recrystallized from pentane; red crystals were obtained. ${ }^{31} \mathrm{P}$ NMR $\left(121.4 \mathrm{MHz}\right.$, benzene- $\left.d_{6}\right): \delta 60.58(\mathrm{~s}) .{ }^{1} \mathrm{H}$ NMR (300 MHz, benzene- $\left.d_{6}\right): 8.11\left(\mathrm{~d}, J_{\mathrm{HH}}=8.1 \mathrm{~Hz}, 1 \mathrm{H}\right.$, nitrophenyl H6), $7.83\left(\mathrm{~d}, J_{\mathrm{HH}}=7.5\right.$ $\mathrm{Hz}, 1 \mathrm{H}$, nitrophenyl H3), 6.94-7.01 (m, 3H, PCP H), $6.93\left(\mathrm{~m}, J_{\mathrm{HH}}=8.1 \mathrm{~Hz}, J_{\mathrm{HH}}=1.2 \mathrm{~Hz}, 1 \mathrm{H}\right.$, nitrophenyl H5), $6.79\left(\mathrm{~m}, J_{\mathrm{HH}}=7.5 \mathrm{~Hz}, J_{\mathrm{HH}}=1.2 \mathrm{~Hz}, 1 \mathrm{H}\right.$, nitrophenyl H4), 3.18 (d of vt, $J_{\mathrm{PH}}=$ $\left.3.0 \mathrm{~Hz}, J_{\mathrm{HH}}=15.6 \mathrm{~Hz}, 2 \mathrm{H}, \mathrm{CH}_{2}\right), 2.94\left(\mathrm{~d}\right.$ of vt, $\left.J_{\mathrm{PH}}=4.2 \mathrm{~Hz}, J_{\mathrm{HH}}=15.6 \mathrm{~Hz}, 2 \mathrm{H}, \mathrm{CH}_{2}\right), 1.30(\mathrm{t}$, $\left.J_{\mathrm{PH}}=6.6 \mathrm{~Hz}, 18 \mathrm{H}, \mathrm{C}\left(\mathrm{CH}_{3}\right)_{3}\right), 0.66\left(\mathrm{t}, J_{\mathrm{PH}}=6.3 \mathrm{~Hz}, 18 \mathrm{H}, \mathrm{C}\left(\mathrm{CH}_{3}\right)_{3}\right),-8.93\left(\mathrm{t}, J_{\mathrm{PH}}=18.2 \mathrm{~Hz}, 1 \mathrm{H}\right.$, Ir- $H)$.

$(\mathbf{P C P}) \operatorname{Ir}(\mathbf{H})\left(\boldsymbol{\kappa}^{\mathbf{2}}-\mathbf{O}, \mathbf{C}-\mathbf{C}_{\mathbf{6}} \mathbf{H}_{\mathbf{4}} \mathbf{N} \mathbf{O}_{2}\right) \mathbf{( 1 \mathbf { b } ) .} 15 \mathrm{mg} \mathbf{1 a}(0.022 \mathrm{mmol})$ was dissolved in $1 \mathrm{~mL} p$ xylene and the solution was then refluxed under argon for 5 hours. Solvent was evacuated and the resulting solid was redissolved in and recrystallized from pentane; red crystals were obtained. ${ }^{31} \mathrm{P}$ NMR (121.4 MHz, benzene- $\left.d_{6}\right): \delta 57.04(\mathrm{~s}) .{ }^{1} \mathrm{H}$ NMR $\left(300 \mathrm{MHz}\right.$, benzene- $\left.d_{6}\right): 8.45\left(\mathrm{~d}, J_{\mathrm{HH}}=\right.$ $7.8 \mathrm{~Hz}, 1 \mathrm{H}$, nitrophenyl H6), 7.28 (d, $J_{\mathrm{HH}}=8.4 \mathrm{~Hz}, 1 \mathrm{H}$, nitrophenyl H3), 7.10-7.25 (m, $3 \mathrm{H}$, PCP H), $7.00\left(\mathrm{~m}, J_{\mathrm{HH}}=7.8 \mathrm{~Hz}, J_{\mathrm{HH}}=0.9 \mathrm{~Hz}, 1 \mathrm{H}\right.$, nitrophenyl H5), $6.74\left(\mathrm{~m}, J_{\mathrm{HH}}=8.4 \mathrm{~Hz}, J_{\mathrm{HH}}\right.$ 
$=1.2 \mathrm{~Hz}, 1 \mathrm{H}$, nitrophenyl H4), 3.43 (d of vt, $J_{\mathrm{PH}}=3.2 \mathrm{~Hz}, J_{\mathrm{HH}}=16.2 \mathrm{~Hz}, 2 \mathrm{H}, \mathrm{CH}$ ), 3.23 (d of vt, $\left.J_{\mathrm{PH}}=4.2 \mathrm{~Hz}, J_{\mathrm{HH}}=16.2 \mathrm{~Hz}, 2 \mathrm{H}, \mathrm{CH}_{2}\right), 1.09\left(\mathrm{t}, J_{\mathrm{PH}}=6.3 \mathrm{~Hz}, 18 \mathrm{H}, \mathrm{C}\left(\mathrm{CH}_{3}\right)_{3}\right), 0.79\left(\mathrm{t}, J_{\mathrm{PH}}=\right.$ $\left.6.3 \mathrm{~Hz}, 18 \mathrm{H}, \mathrm{C}\left(\mathrm{CH}_{3}\right)_{3}\right),-24.84\left(\mathrm{t}, J_{\mathrm{PH}}=15.5 \mathrm{~Hz}, 1 \mathrm{H}, \mathrm{Ir}-H\right)$.

(PCP)IrH( $\left.\boldsymbol{\kappa}^{2}-\mathbf{O}, \mathbf{C}-\mathbf{C}_{6} \mathbf{H}_{4} \mathbf{C}(\mathbf{O}) \mathbf{C H}_{3}\right)$ (2a). $20 \mathrm{mg}(\mathrm{PCP}) \mathrm{IrH}_{2}(0.034 \mathrm{mmol})$ was dissolved in $2 \mathrm{~mL} p$-xylene solution containing $5.0 \mathrm{mg}$ norbornene $(0.053 \mathrm{mmol})$. To the resulting solution was added $5.1 \mu \mathrm{L}$ acetophenone $(0.052 \mathrm{mmol})$; after stirring for ca. $1 \mathrm{~min}$, the red solution turned orange. Solvent was evacuated and the resulting solid was redissolved in and recrystallized from pentane; red crystals were obtained. ${ }^{31} \mathrm{P}$ NMR $\left(121.4 \mathrm{MHz}\right.$, benzene- $\left.d_{6}\right): \delta 57.05$ (s). ${ }^{1} \mathrm{H}$ NMR (300 MHz, benzene- $\left.d_{6}\right): 7.91\left(\mathrm{~d} J_{\mathrm{HH}}=7.5 \mathrm{~Hz}, 1 \mathrm{H}\right.$, acetylphenyl H6), $7.58\left(\mathrm{~d}, J_{\mathrm{HH}}=8.1 \mathrm{~Hz}, 1 \mathrm{H}\right.$, acetylphenyl H3), 7.20-7.01 (m, 3 H, PCP H), $6.97\left(\mathrm{dt}, J_{\mathrm{HH}}=7.5 \mathrm{~Hz}, J_{\mathrm{HH}}=1.2 \mathrm{~Hz}, 1 \mathrm{H}\right.$, acetylphenyl H5), $6.86\left(\mathrm{dt}, J_{\mathrm{HH}}=8.1 \mathrm{~Hz}, J_{\mathrm{HH}}=0.9 \mathrm{~Hz}, 1 \mathrm{H}\right.$, acetylphenyl H4), $3.21\left(\mathrm{~d}\right.$ of vt, $J_{\mathrm{PH}}$ $\left.=3.0 \mathrm{~Hz}, J_{\mathrm{HH}}=15.6 \mathrm{~Hz}, 2 \mathrm{H}, \mathrm{CH}_{2}\right), 2.93\left(\mathrm{~d}\right.$ of vt, $\left.J_{\mathrm{PH}}=4.1 \mathrm{~Hz}, J_{\mathrm{HH}}=15.6 \mathrm{~Hz}, 2 \mathrm{H}, \mathrm{CH}_{2}\right), 2.37$ $\left(\mathrm{s}, 3 \mathrm{H}, \mathrm{CH}_{3}\right), 1.33\left(\mathrm{t}, J_{\mathrm{PH}}=6.30 \mathrm{~Hz}, 18 \mathrm{H}, \mathrm{C}\left(\mathrm{CH}_{3}\right)_{3}\right), 0.66\left(\mathrm{t}, J_{\mathrm{PH}}=6.0 \mathrm{~Hz}, 18 \mathrm{H}, \mathrm{C}\left(\mathrm{CH}_{3}\right)_{3}\right)$, $-8.93\left(\mathrm{t}, J_{\mathrm{PH}}=18.6 \mathrm{~Hz}, 1 \mathrm{H}, \mathrm{Ir}-H\right)$. IR (pentane): $v_{\mathrm{CO}} 1567.8 \mathrm{~cm}^{-1}$.

(PCP)IrH( $\left.\left.\boldsymbol{\kappa}^{2}-\mathbf{O}, \mathbf{C}-\mathbf{C}_{6} \mathbf{H}_{4} \mathbf{C}(\mathbf{O}) \mathbf{C H}_{3}\right) \mathbf{( 2 b}\right) .15 \mathrm{mg} \mathbf{2 a}(0.022 \mathrm{mmol})$ was dissolved in $1 \mathrm{~mL}$ $p$-xylene. The solution was then refluxed under argon for 5 hours. Solvent was evacuated and the resulting solid was redissolved in and recrystallized from pentane; red crystals were obtained. ${ }^{31} \mathrm{P}$ NMR (121.4 MHz, benzene- $\left.d_{6}\right): \delta 53.99$ (s). ${ }^{1} \mathrm{H}$ NMR (300 MHz, benzene- $\left.d_{6}\right): 8.57$ (d, $J_{\mathrm{HH}}=$ $7.5 \mathrm{~Hz}, 1 \mathrm{H}$, acetylphenyl H6), $7.65\left(\mathrm{~d}, J_{\mathrm{HH}}=7.2 \mathrm{~Hz}, 1 \mathrm{H}\right.$, acetylphenyl H3), 7.20-7.29 (m, $3 \mathrm{H}$, PCP H), $7.11\left(\mathrm{dt}, J_{\mathrm{HH}}=7.5 \mathrm{~Hz}, J_{\mathrm{HH}}=1.5 \mathrm{~Hz}, 1 \mathrm{H}\right.$, acetylphenyl H5), $6.89\left(\mathrm{dt}, J_{\mathrm{HH}}=7.2 \mathrm{~Hz}, J_{\mathrm{HH}}\right.$ $=1.5 \mathrm{~Hz}, 1 \mathrm{H}$, acetylphenyl H4), $3.49\left(\mathrm{~d}\right.$ of vt, $\left.J_{\mathrm{PH}}=3.0 \mathrm{~Hz}, J_{\mathrm{HH}}=16.2 \mathrm{~Hz}, 2 \mathrm{H}, \mathrm{CH}_{2}\right), 3.26(\mathrm{~d}$ of vt, $\left.J_{\mathrm{PH}}=4.2 \mathrm{~Hz}, J_{\mathrm{HH}}=16.2 \mathrm{~Hz}, 2 \mathrm{H}, \mathrm{CH}_{2}\right), 2.40\left(\mathrm{~s}, 3 \mathrm{H}, \mathrm{CH}_{3}\right), 1.15\left(\mathrm{t}, J_{\mathrm{PH}}=6.15 \mathrm{~Hz}, 18 \mathrm{H}\right.$, $\left.\mathrm{C}\left(\mathrm{CH}_{3}\right)_{3}\right), 0.80\left(\mathrm{t}, J_{\mathrm{PH}}=6.15 \mathrm{~Hz}, 18 \mathrm{H}, \mathrm{C}\left(\mathrm{CH}_{3}\right)_{3}\right),-24.68\left(\mathrm{t}, J_{\mathrm{PH}}=16.2 \mathrm{~Hz}, 1 \mathrm{H}, \mathrm{Ir}-H\right) . \mathrm{IR}$ (pentane): $v_{\mathrm{CO}} 1572.6 \mathrm{~cm}^{-1}$.

\section{Low temperature observation of (PCP)Ir(3,5-dimethyl-4-nitrophenyl)(H) (3) (para} and meta isomer/rotamers). Into a small vial was placed $10 \mathrm{mg}(\mathrm{PCP}) \mathrm{IrH}_{2}(0.017 \mathrm{mmol})$ followed by $0.3 \mathrm{~mL}$ of mesitylene- $d_{12}$ solution containing 2 eq norbornene. The mixed solution was transferred to a 5-mm extended NMR tube. The tube was then cooled to $-78{ }^{\circ} \mathrm{C}$, and $4.4 \mu \mathrm{L}$ nitrobenzene $(0.043 \mathrm{mmol})$ in $0.2 \mathrm{ml}$ mesitylene- $d_{12}$ was slowly added by syringe. The tube was sealed under argon protection and then inserted into a pre-cooled NMR spectrometer probe, 
allowed to warm up to $-47^{\circ} \mathrm{C}$, and monitored by NMR. After $40 \mathrm{~min}$, the starting material had undergone $85 \%$ conversion to the products assigned as isomers/rotamers of $3 .{ }^{31} \mathrm{P}$ NMR (selectively proton-decoupled; mesitylene- $d_{12},-47{ }^{\circ} \mathrm{C}$ ): $\delta 67.28$ (d), 66.67 (d), 66.17 (d); ${ }^{1} \mathrm{H}$ NMR (mesitylene- $\left.d_{12},-47{ }^{\circ} \mathrm{C}\right):-45.12(\mathrm{t}),-45.20(\mathrm{t}),-45.52(\mathrm{t}) \mathrm{ppm}$. Other ${ }^{1} \mathrm{H}$ NMR product peaks could not be characterized due to overlap with excess norbornene and norbornane peaks. Upon allowing the solution to warm, complete conversion to $1 \mathrm{a}$ occurred at $-10{ }^{\circ} \mathrm{C}$ after $40 \mathrm{~min}$.

Low temperature observation of (PCP)Ir(3,5-dimethyl-4-nitrophenyl)(H) (3) with 4d-nitrobenzene. The same procedure as above was followed but with 2.5 eq 4-d-norbornene. After $40 \mathrm{~min}$ at $-47^{\circ} \mathrm{C}$, the starting material was converted to the reaction intermediates, $90 \%$ (NMR). ${ }^{31} \mathrm{P}$ NMR (mesitylene- $d_{12},-47{ }^{\circ} \mathrm{C}$ ): $\delta 67.17$ (s), 66.53 (d), 66.10 (d); ${ }^{1} \mathrm{H}$ NMR (mesitylene- $d_{12},-47^{\circ} \mathrm{C}$ ): $-45.20(\mathrm{t}),-45.52(\mathrm{t}) \mathrm{ppm}$. Other ${ }^{1} \mathrm{H}$ NMR product peaks could not be characterized due to overlap with excess norbornene and norbornane peaks. Upon allowing the solution to warm, complete conversion to $1 \mathrm{a}$ occurred at $-10{ }^{\circ} \mathrm{C}$ after $40 \mathrm{~min}$.

X-ray Structure Determination of 1a, 1b, 2a, 2b. Data for 1a, 1b, 2a, 2b were collected on a Bruker Smart APEX CCD diffractometer with graphite monochromatized Mo K $\alpha$ radiation $(\lambda=0.71073 \AA)$ at $100 \mathrm{~K}$. The data were corrected for Lorenz effects and polarization, and absorption, the latter by a multi-scan (SADABS) $)^{3}$ method. The structures were solved by Patterson or direct methods (SHELXS86). ${ }^{4}$ All non-hydrogen atoms were refined (SHELXL97) ${ }^{5}$ based upon $\mathrm{F}_{\mathrm{obs}}^{2}$. All hydrogen atom coordinates were calculated with idealized geometries (SHELXL97). Scattering factors $\left(f_{0}, f^{\prime}, f^{\prime \prime}\right)$ are as described in SHELXL97.

\section{References}

(1) Gupta, M.; Hagen, C.; Flesher, R. J.; Kaska, W. C.; Jensen, C. M. Chem. Comm. 1996, 2083-2084.

(2) Makosza, M.; Stalinski, K. Tetrahedron Lett. 1998, 39, 3575-3576.

(3) Bruker-AXS. SADABS, Bruker Nonius area detector scaling and absorption correction, v2.05, Bruker-AXS Inc., Madison, Wisconsin, 2003.

(4) Sheldrick, G. M. SHELXS86, Program for the Solution of Crystal Structures, University of Göttingen, Germany, 1986.

(5) Sheldrick, G. M. SHELXL97, Program for Crystal Structure Refinement, University of Göttingen, Germany, 1997. 


\section{Structural Data for 1a}
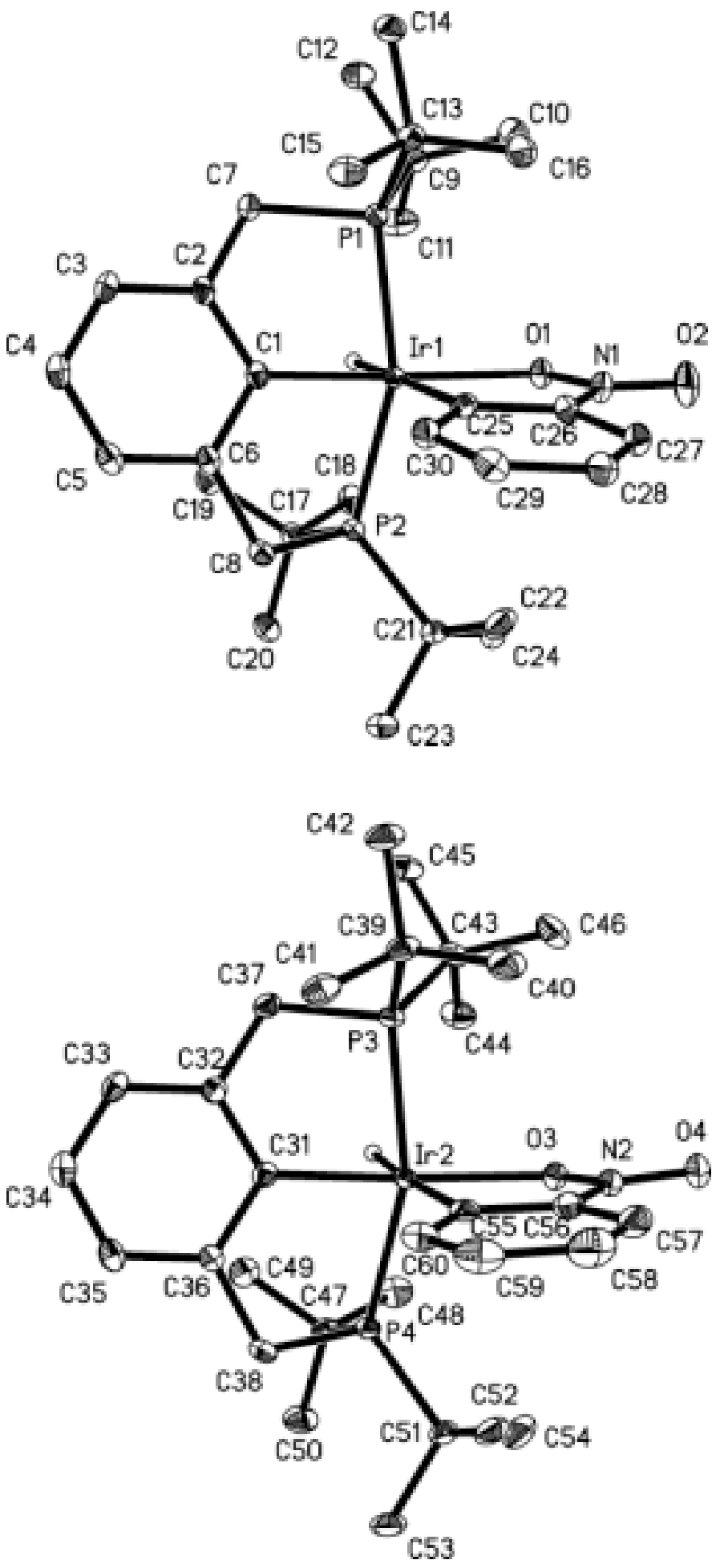

Figure S-1. ORTEP diagrams of 1a. Hydrogen atoms omitted for clarity. Thermal ellipsoids are shown at $50 \%$ probability. 
Table S-1. Crystal data and structure refinement for $\mathbf{1 a}$.

Identification code

Empirical formula

Formula weight

Temperature

Wavelength

Crystal system

Space group

Unit cell dimensions

Volume

Z

Density (calculated)

Absorption coefficient

$\mathrm{F}(000)$

Crystal size

Theta range for data collection

Index ranges

Reflections collected

Independent reflections

Completeness to theta $=30.51^{\circ}$

Absorption correction

Max. and min. transmission

Refinement method

Data / restraints / parameters

Goodness-of-fit on $\mathrm{F}^{2}$

Final $\mathrm{R}$ indices [I $>2 \operatorname{sigma}(\mathrm{I})]$

$\mathrm{R}$ indices (all data)

Largest diff. peak and hole irhnobenz

C30 H48 Ir N O2 P2

708.83

$100(2) \mathrm{K}$

$0.71073 \AA$

Monoclinic

$\mathrm{P} 2(1) / \mathrm{c}$

$\mathrm{a}=16.0055(9) \AA$

$\alpha=90^{\circ}$.

$\mathrm{b}=11.2575(6) \AA$

$\mathrm{c}=34.1472(19) \AA$

6036.5(6) $\AA^{3}$

8

$1.560 \mathrm{Mg} / \mathrm{m}^{3}$

$4.556 \mathrm{~mm}^{-1}$

2864

$0.25 \times 0.20 \times 0.03 \mathrm{~mm}^{3}$

1.22 to $30.51^{\circ}$.

$-22<=\mathrm{h}<=22,-16<=\mathrm{k}<=16,-48<=\mathrm{l}<=48$

122334

$18421[\mathrm{R}(\mathrm{int})=0.0429]$

$100.0 \%$

Semi-empirical from equivalents

0.8754 and 0.3955

Full-matrix least-squares on $\mathrm{F}^{2}$

$18421 / 2$ / 681

1.003

$\mathrm{R} 1=0.0253, \mathrm{wR} 2=0.0543$

$\mathrm{R} 1=0.0303, \mathrm{wR} 2=0.0559$

1.486 and -1.167 e. $\AA^{-3}$ 
Table S-2. Atomic coordinates ( x 10 $0^{4}$ ) and equivalent isotropic displacement parameters $\left(\AA^{2} \times 10^{3}\right)$ for 1a. $\mathrm{U}(\mathrm{eq})$ is defined as one third of the trace of the orthogonalized $\mathrm{U}^{\mathrm{ij}}$ tensor.

\begin{tabular}{|c|c|c|c|c|}
\hline & $\mathrm{x}$ & $\mathrm{y}$ & $\mathrm{Z}$ & $\bar{U}(\mathrm{eq})$ \\
\hline $\operatorname{Ir}(1)$ & $4175(1)$ & $5182(1)$ & $1397(1)$ & $10(1)$ \\
\hline $\mathrm{P}(1)$ & $4952(1)$ & $6764(1)$ & $1211(1)$ & $11(1)$ \\
\hline $\mathrm{P}(2)$ & $3660(1)$ & $3900(1)$ & $1833(1)$ & $12(1)$ \\
\hline $\mathrm{C}(1)$ & $5278(2)$ & $4832(2)$ & $1783(1)$ & $12(1)$ \\
\hline $\mathrm{C}(2)$ & $5962(2)$ & $5648(2)$ & $1844(1)$ & $13(1)$ \\
\hline $\mathrm{C}(3)$ & $6733(2)$ & $5386(2)$ & 2098(1) & $16(1)$ \\
\hline C(4) & $6828(2)$ & $4334(2)$ & $2317(1)$ & $17(1)$ \\
\hline$C(5)$ & $6148(2)$ & $3551(2)$ & $2282(1)$ & $16(1)$ \\
\hline$C(6)$ & $5382(2)$ & $3783(2)$ & $2018(1)$ & $13(1)$ \\
\hline$C(7)$ & $5804(2)$ & $6861(2)$ & $1653(1)$ & $14(1)$ \\
\hline $\mathrm{C}(8)$ & $4615(2)$ & $2988(2)$ & $2002(1)$ & $15(1)$ \\
\hline $\mathrm{C}(9)$ & $4445(2)$ & $8296(2)$ & $1179(1)$ & $16(1)$ \\
\hline$C(10)$ & $3887(2)$ & $8494(3)$ & $764(1)$ & $28(1)$ \\
\hline$C(11)$ & $3863(2)$ & $8396(3)$ & $1488(1)$ & $26(1)$ \\
\hline$C(12)$ & $5106(2)$ & $9308(2)$ & $1274(1)$ & $21(1)$ \\
\hline$C(13)$ & $5547(2)$ & $6576(2)$ & $788(1)$ & $15(1)$ \\
\hline$C(14)$ & $6080(2)$ & $7659(2)$ & $712(1)$ & $18(1)$ \\
\hline$C(15)$ & $6171(2)$ & $5538(2)$ & $908(1)$ & $20(1)$ \\
\hline$C(16)$ & $4933(2)$ & $6231(3)$ & 401(1) & 21(1) \\
\hline$C(17)$ & $3458(2)$ & $4682(2)$ & $2300(1)$ & $14(1)$ \\
\hline $\mathrm{C}(18)$ & $2801(2)$ & $5683(3)$ & $2189(1)$ & $18(1)$ \\
\hline C(19) & $4293(2)$ & $5240(2)$ & $2522(1)$ & $17(1)$ \\
\hline$C(20)$ & $3133(2)$ & $3849(3)$ & $2593(1)$ & 21(1) \\
\hline $\mathrm{C}(21)$ & $2785(2)$ & $2757(2)$ & $1675(1)$ & $16(1)$ \\
\hline $\mathrm{C}(22)$ & $2873(2)$ & $2243(3)$ & $1268(1)$ & $24(1)$ \\
\hline $\mathrm{C}(23)$ & $2837(2)$ & $1693(3)$ & $1962(1)$ & $25(1)$ \\
\hline $\mathrm{C}(24)$ & $1903(2)$ & $3337(3)$ & $1624(1)$ & $23(1)$ \\
\hline $\mathrm{N}(1)$ & $2978(1)$ & $4817(2)$ & $627(1)$ & $16(1)$ \\
\hline $\mathrm{O}(1)$ & $3055(1)$ & $5497(2)$ & $928(1)$ & $16(1)$ \\
\hline $\mathrm{O}(2)$ & 2381(1) & $4942(2)$ & $342(1)$ & $28(1)$ \\
\hline$C(25)$ & $4263(2)$ & $3889(2)$ & $968(1)$ & $13(1)$ \\
\hline$C(26)$ & $3600(2)$ & $3889(2)$ & $635(1)$ & $13(1)$ \\
\hline $\mathrm{C}(27)$ & $3468(2)$ & $3042(2)$ & $329(1)$ & $17(1)$ \\
\hline $\mathrm{C}(28)$ & $4045(2)$ & $2130(2)$ & $352(1)$ & $19(1)$ \\
\hline C(29) & $4745(2)$ & 2091(2) & 674(1) & $18(1)$ \\
\hline $\mathrm{C}(30)$ & $4849(2)$ & $2944(2)$ & $973(1)$ & $15(1)$ \\
\hline $\operatorname{Ir}(2)$ & $867(1)$ & $4683(1)$ & $3864(1)$ & $10(1)$ \\
\hline $\mathrm{P}(3)$ & $20(1)$ & $3149(1)$ & $4025(1)$ & $12(1)$ \\
\hline $\mathrm{P}(4)$ & $1641(1)$ & $6442(1)$ & $3939(1)$ & $12(1)$ \\
\hline $\mathrm{C}(31)$ & $77(2)$ & $5691(2)$ & $4125(1)$ & $12(1)$ \\
\hline$C(32)$ & $-468(2)$ & $5169(2)$ & $4357(1)$ & $14(1)$ \\
\hline$C(33)$ & $-1025(2)$ & $5868(2)$ & $4530(1)$ & $16(1)$ \\
\hline$C(34)$ & $-1023(2)$ & $7098(3)$ & 4491(1) & $18(1)$ \\
\hline$C(35)$ & $-452(2)$ & $7634(2)$ & $4289(1)$ & $16(1)$ \\
\hline$C(36)$ & $95(2)$ & $6944(2)$ & $4110(1)$ & $12(1)$ \\
\hline$C(37)$ & $-395(2)$ & $3845(2)$ & $4435(1)$ & $15(1)$ \\
\hline $\mathrm{C}(38)$ & $771(2)$ & $7520(2)$ & $3921(1)$ & $17(1)$ \\
\hline C(39) & $-982(2)$ & $2715(2)$ & $3664(1)$ & $17(1)$ \\
\hline$C(40)$ & $-800(2)$ & $2323(3)$ & $3257(1)$ & $23(1)$ \\
\hline$C(41)$ & $-1540(2)$ & $3839(3)$ & $3595(1)$ & $21(1)$ \\
\hline$C(42)$ & $-1496(2)$ & $1740(3)$ & $3826(1)$ & $23(1)$ \\
\hline$C(43)$ & $599(2)$ & $1766(2)$ & $4246(1)$ & $18(1)$ \\
\hline$C(44)$ & $1493(2)$ & $2109(3)$ & $4471(1)$ & $22(1)$ \\
\hline$C(45)$ & $150(2)$ & $1130(3)$ & $4549(1)$ & $24(1)$ \\
\hline$C(46)$ & $723(2)$ & $905(3)$ & $3912(1)$ & $27(1)$ \\
\hline$C(47)$ & $2336(2)$ & $6616(2)$ & $4456(1)$ & $17(1)$ \\
\hline $\mathrm{C}(48)$ & $2958(2)$ & $5557(3)$ & $4543(1)$ & $23(1)$ \\
\hline C(49) & $1757(2)$ & $6590(3)$ & $4769(1)$ & $19(1)$ \\
\hline$C(50)$ & $2846(2)$ & $7777(3)$ & $4524(1)$ & $24(1)$ \\
\hline
\end{tabular}




\begin{tabular}{|c|c|c|c|c|}
\hline$C(51)$ & $2239(2)$ & $7025(2)$ & $3551(1)$ & $17(1)$ \\
\hline$C(52)$ & $1722(2)$ & $6782(3)$ & $3130(1)$ & $22(1)$ \\
\hline$C(53)$ & $2378(2)$ & $8380(3)$ & $3579(1)$ & $27(1)$ \\
\hline$C(54)$ & $3091(2)$ & $6374(3)$ & $3585(1)$ & $26(1)$ \\
\hline $\mathrm{N}(2)$ & $1396(2)$ & $3401(2)$ & $3197(1)$ & $17(1)$ \\
\hline $\mathrm{O}(3)$ & $1656(1)$ & $3532(2)$ & $3572(1)$ & $16(1)$ \\
\hline $\mathrm{O}(4)$ & $1740(1)$ & $2660(2)$ & $3012(1)$ & $26(1)$ \\
\hline$C(55)$ & $346(2)$ & $4873(2)$ & $3259(1)$ & $14(1)$ \\
\hline$C(56)$ & $706(2)$ & $4137(2)$ & $3004(1)$ & $17(1)$ \\
\hline$C(57)$ & $456(2)$ & $4053(3)$ & $2588(1)$ & $24(1)$ \\
\hline$C(58)$ & $-199(2)$ & $4772(3)$ & $2409(1)$ & $31(1)$ \\
\hline$C(59)$ & $-591(2)$ & $5544(3)$ & $2642(1)$ & $27(1)$ \\
\hline$C(60)$ & $-326(2)$ & $5593(2)$ & $3054(1)$ & $19(1)$ \\
\hline
\end{tabular}

Table S-3. Selected Bond lengths $[\AA]$ and angles $\left[{ }^{\circ}\right]$ for $\mathbf{1 a}$.

\begin{tabular}{|c|c|c|c|}
\hline $\operatorname{Ir}(1)-C(1)$ & $2.027(2)$ & $\operatorname{Ir}(2)-C(31)$ & $2.028(2)$ \\
\hline $\operatorname{Ir}(1)-C(25)$ & $2.087(2)$ & $\operatorname{Ir}(2)-C(55)$ & $2.084(2)$ \\
\hline $\operatorname{Ir}(1)-\mathrm{O}(1)$ & $2.1883(18)$ & $\operatorname{Ir}(2)-\mathrm{O}(3)$ & $2.1824(18)$ \\
\hline $\operatorname{Ir}(1)-P(1)$ & $2.3292(6)$ & $\operatorname{Ir}(2)-P(4)$ & $2.3239(6)$ \\
\hline $\operatorname{Ir}(1)-\mathrm{P}(2)$ & $2.3363(6)$ & $\operatorname{Ir}(2)-\mathrm{P}(3)$ & $2.3251(6)$ \\
\hline $\operatorname{Ir}(1)-\mathrm{H}(1)$ & $1.590(10)$ & $\operatorname{Ir}(2)-\mathrm{H}(2)$ & $1.593(10)$ \\
\hline $\mathrm{N}(1)-\mathrm{O}(2)$ & $1.233(3)$ & $\mathrm{N}(2)-\mathrm{O}(4)$ & $1.238(3)$ \\
\hline $\mathrm{N}(1)-\mathrm{O}(1)$ & $1.268(3)$ & $\mathrm{N}(2)-\mathrm{O}(3)$ & $1.274(3)$ \\
\hline $\mathrm{N}(1)-\mathrm{C}(26)$ & $1.440(3)$ & $\mathrm{N}(2)-\mathrm{C}(56)$ & $1.436(4)$ \\
\hline$C(25)-C(26)$ & $1.396(3)$ & $\mathrm{C}(55)-\mathrm{C}(56)$ & $1.405(4)$ \\
\hline $\mathrm{C}(25)-\mathrm{C}(30)$ & $1.417(3)$ & $\mathrm{C}(55)-\mathrm{C}(60)$ & $1.419(4)$ \\
\hline$C(26)-C(27)$ & $1.401(3)$ & $\mathrm{C}(56)-\mathrm{C}(57)$ & $1.400(4)$ \\
\hline C(27)-C(28) & $1.373(4)$ & $\mathrm{C}(57)-\mathrm{C}(58)$ & $1.372(5)$ \\
\hline $\mathrm{C}(28)-\mathrm{C}(29)$ & $1.410(4)$ & $\mathrm{C}(58)-\mathrm{C}(59)$ & $1.405(5)$ \\
\hline $\mathrm{C}(29)-\mathrm{C}(30)$ & $1.388(4)$ & $\mathrm{C}(59)-\mathrm{C}(60)$ & $1.391(4)$ \\
\hline$C(1)-\operatorname{Ir}(1)-C(25)$ & $98.80(10)$ & $C(31)-\operatorname{Ir}(2)-C(55)$ & $102.35(10)$ \\
\hline $\mathrm{C}(1)-\operatorname{Ir}(1)-\mathrm{O}(1)$ & $173.83(8)$ & $\mathrm{C}(31)-\operatorname{Ir}(2)-\mathrm{O}(3)$ & $176.79(9)$ \\
\hline$C(25)-\operatorname{Ir}(1)-\mathrm{O}(1)$ & $75.95(8)$ & $\mathrm{C}(55)-\operatorname{Ir}(2)-\mathrm{O}(3)$ & $76.29(9)$ \\
\hline $\mathrm{C}(1)-\operatorname{Ir}(1)-\mathrm{P}(1)$ & $83.27(7)$ & $\mathrm{C}(31)-\operatorname{Ir}(2)-\mathrm{P}(4)$ & $80.75(7)$ \\
\hline$C(25)-\operatorname{Ir}(1)-P(1)$ & $103.33(7)$ & $\mathrm{C}(55)-\operatorname{Ir}(2)-\mathrm{P}(4)$ & $97.10(7)$ \\
\hline $\mathrm{O}(1)-\operatorname{Ir}(1)-\mathrm{P}(1)$ & $94.70(5)$ & $\mathrm{O}(3)-\operatorname{Ir}(2)-\mathrm{P}(4)$ & $102.27(5)$ \\
\hline $\mathrm{C}(1)-\operatorname{Ir}(1)-\mathrm{P}(2)$ & $80.49(7)$ & $\mathrm{C}(31)-\operatorname{Ir}(2)-\mathrm{P}(3)$ & $82.58(7)$ \\
\hline $\mathrm{C}(25)-\operatorname{Ir}(1)-\mathrm{P}(2)$ & $95.52(7)$ & $\mathrm{C}(55)-\operatorname{Ir}(2)-\mathrm{P}(3)$ & $99.85(7)$ \\
\hline $\mathrm{O}(1)-\operatorname{Ir}(1)-\mathrm{P}(2)$ & $103.06(5)$ & $\mathrm{O}(3)-\operatorname{Ir}(2)-\mathrm{P}(3)$ & $94.75(5)$ \\
\hline $\mathrm{P}(1)-\operatorname{Ir}(1)-\mathrm{P}(2)$ & $156.76(2)$ & $\mathrm{P}(4)-\operatorname{Ir}(2)-\mathrm{P}(3)$ & $158.38(2)$ \\
\hline $\mathrm{C}(1)-\operatorname{Ir}(1)-\mathrm{H}(1)$ & $87.2(12)$ & $\mathrm{C}(31)-\operatorname{Ir}(2)-\mathrm{H}(2)$ & $82.5(16)$ \\
\hline C(25)-Ir(1)-H(1) & $173.2(12)$ & $\mathrm{C}(55)-\operatorname{Ir}(2)-\mathrm{H}(2)$ & $174.4(16)$ \\
\hline $\mathrm{O}(1)-\operatorname{Ir}(1)-\mathrm{H}(1)$ & $98.2(12)$ & $\mathrm{O}(3)-\operatorname{Ir}(2)-\mathrm{H}(2)$ & $98.8(16)$ \\
\hline $\mathrm{P}(1)-\operatorname{Ir}(1)-\mathrm{H}(1)$ & $80.5(12)$ & $\mathrm{P}(4)-\operatorname{Ir}(2)-\mathrm{H}(2)$ & $86.4(16)$ \\
\hline $\mathrm{P}(2)-\operatorname{Ir}(1)-\mathrm{H}(1)$ & $82.2(12)$ & $\mathrm{P}(3)-\operatorname{Ir}(2)-\mathrm{H}(2)$ & $77.8(16)$ \\
\hline $\mathrm{O}(2)-\mathrm{N}(1)-\mathrm{O}(1)$ & $120.7(2)$ & $\mathrm{O}(4)-\mathrm{N}(2)-\mathrm{O}(3)$ & $119.9(2)$ \\
\hline $\mathrm{O}(2)-\mathrm{N}(1)-\mathrm{C}(26)$ & $121.7(2)$ & $\mathrm{O}(4)-\mathrm{N}(2)-\mathrm{C}(56)$ & $122.2(2)$ \\
\hline $\mathrm{O}(1)-\mathrm{N}(1)-\mathrm{C}(26)$ & $117.6(2)$ & $\mathrm{O}(3)-\mathrm{N}(2)-\mathrm{C}(56)$ & $118.0(2)$ \\
\hline $\mathrm{N}(1)-\mathrm{O}(1)-\operatorname{Ir}(1)$ & $115.60(15)$ & $\mathrm{N}(2)-\mathrm{O}(3)-\operatorname{Ir}(2)$ & $114.96(15)$ \\
\hline $\mathrm{C}(26)-\mathrm{C}(25)-\mathrm{C}(30)$ & $113.9(2)$ & $\mathrm{C}(56)-\mathrm{C}(55)-\mathrm{C}(60)$ & $113.1(2)$ \\
\hline$C(26)-C(25)-\operatorname{Ir}(1)$ & $115.29(18)$ & $C(56)-C(55)-\operatorname{Ir}(2)$ & $114.97(19)$ \\
\hline $\mathrm{C}(30)-\mathrm{C}(25)-\operatorname{Ir}(1)$ & $130.55(18)$ & $\mathrm{C}(60)-\mathrm{C}(55)-\operatorname{Ir}(2)$ & $131.9(2)$ \\
\hline$C(25)-C(26)-C(27)$ & 126.1(2) & $\mathrm{C}(57)-\mathrm{C}(56)-\mathrm{C}(55)$ & $126.8(3)$ \\
\hline $\mathrm{C}(25)-\mathrm{C}(26)-\mathrm{N}(1)$ & $115.4(2)$ & $\mathrm{C}(57)-\mathrm{C}(56)-\mathrm{N}(2)$ & $118.1(2)$ \\
\hline $\mathrm{C}(27)-\mathrm{C}(26)-\mathrm{N}(1)$ & $118.4(2)$ & $\mathrm{C}(55)-\mathrm{C}(56)-\mathrm{N}(2)$ & $115.2(2)$ \\
\hline
\end{tabular}




\section{Structural Data for $1 \mathrm{~b}$}

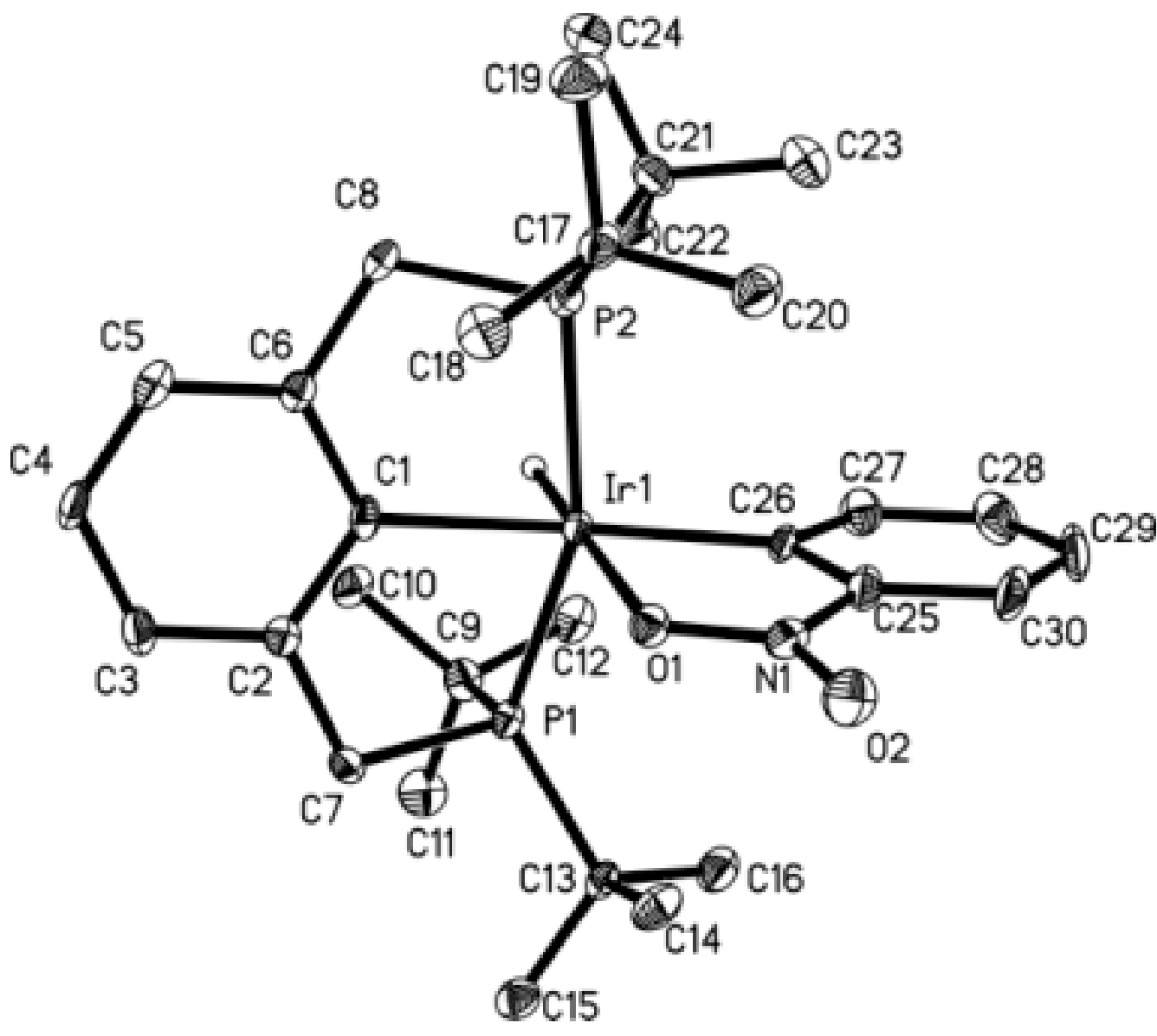

Figure S-2. ORTEP diagram of 1b. Hydrogen atoms omitted for clarity. Thermal ellipsoids are shown at $50 \%$ probability. 
Table S-4. Crystal data and structure refinement for $\mathbf{1 b}$.

\begin{tabular}{|c|c|c|}
\hline Identification code & \multicolumn{2}{|l|}{ irhboo } \\
\hline Empirical formula & \multicolumn{2}{|l|}{ C30 H48 Ir N O2 P2 } \\
\hline Formula weight & \multicolumn{2}{|l|}{708.83} \\
\hline Temperature & \multicolumn{2}{|l|}{$100(2) \mathrm{K}$} \\
\hline Wavelength & \multicolumn{2}{|l|}{$0.71073 \AA$} \\
\hline Crystal system & \multicolumn{2}{|l|}{ Monoclinic } \\
\hline Space group & \multicolumn{2}{|l|}{$\mathrm{P} 2(1) / \mathrm{a}$} \\
\hline \multirow[t]{3}{*}{ Unit cell dimensions } & $\mathrm{a}=12.3806(11) \AA$ & $\alpha=90^{\circ}$ \\
\hline & $\mathrm{b}=17.1783(16) \AA$ & $\beta=94.535(2)^{\circ}$ \\
\hline & $\mathrm{c}=14.0018(13) \AA$ & $\gamma=90^{\circ}$. \\
\hline Volume & \multicolumn{2}{|l|}{$2968.5(5) \AA^{3}$} \\
\hline $\mathrm{Z}$ & \multicolumn{2}{|l|}{4} \\
\hline Density (calculated) & \multicolumn{2}{|l|}{$1.586 \mathrm{Mg} / \mathrm{m}^{3}$} \\
\hline Absorption coefficient & \multicolumn{2}{|l|}{$4.633 \mathrm{~mm}^{-1}$} \\
\hline $\mathrm{F}(000)$ & \multicolumn{2}{|l|}{1432} \\
\hline Crystal size & \multicolumn{2}{|c|}{$0.07 \times 0.06 \times 0.03 \mathrm{~mm}^{3}$} \\
\hline Theta range for data collection & \multicolumn{2}{|l|}{1.88 to $30.67^{\circ}$. } \\
\hline Index ranges & \multicolumn{2}{|c|}{$-17<=\mathrm{h}<=17,-24<=\mathrm{k}<=24,-20<=1<=19$} \\
\hline Reflections collected & \multicolumn{2}{|l|}{35123} \\
\hline Independent reflections & \multicolumn{2}{|c|}{$9123[\mathrm{R}(\mathrm{int})=0.0745]$} \\
\hline Completeness to theta $=30.67^{\circ}$ & \multicolumn{2}{|l|}{$99.2 \%$} \\
\hline Absorption correction & \multicolumn{2}{|c|}{ Semi-empirical from equivalents } \\
\hline Max. and min. transmission & \multicolumn{2}{|l|}{0.8930 and 0.7375} \\
\hline Refinement method & \multicolumn{2}{|c|}{ Full-matrix least-squares on $\mathrm{F}^{2}$} \\
\hline Data / restraints / parameters & \multicolumn{2}{|l|}{$9123 / 1 / 340$} \\
\hline Goodness-of-fit on $\mathrm{F}^{2}$ & \multicolumn{2}{|l|}{1.008} \\
\hline Final $\mathrm{R}$ indices $[\mathrm{I}>2 \operatorname{sigma}(\mathrm{I})]$ & \multicolumn{2}{|c|}{$\mathrm{R} 1=0.0452, \mathrm{wR} 2=0.0970$} \\
\hline $\mathrm{R}$ indices (all data) & \multicolumn{2}{|c|}{$\mathrm{R} 1=0.0808, \mathrm{wR} 2=0.1110$} \\
\hline Largest diff. peak and hole & \multicolumn{2}{|c|}{5.508 and -2.461 e. $\AA^{-3}$} \\
\hline
\end{tabular}


Table S-5. Atomic coordinates $\left(\times 10^{4}\right)$ and equivalent isotropic displacement parameters $\left(\AA^{2} \times 10^{3}\right)$ for $\mathbf{1 b}$. $\mathrm{U}(\mathrm{eq})$ is defined as one third of the trace of the orthogonalized $\mathrm{U}^{\mathrm{ij}}$ tensor.

\begin{tabular}{|c|c|c|c|c|}
\hline & $\mathrm{x}$ & $y$ & $z$ & $\mathrm{U}(\mathrm{eq})$ \\
\hline $\operatorname{Ir}(1)$ & 1862(1) & $-15(1)$ & 2339(1) & 11(1) \\
\hline $\mathrm{P}(1)$ & $1235(1)$ & $1140(1)$ & $1648(1)$ & $13(1)$ \\
\hline $\mathrm{P}(2)$ & 2014(1) & $-1019(1)$ & 3451(1) & $14(1)$ \\
\hline$C(1)$ & $244(4)$ & $-44(3)$ & 2691(3) & $14(1)$ \\
\hline$C(2)$ & $-561(4)$ & $403(3)$ & 2188(4) & $13(1)$ \\
\hline$C(3)$ & $-1643(4)$ & $333(3)$ & 2384(4) & $18(1)$ \\
\hline C(4) & $-1941(4)$ & $-172(3)$ & 3098(4) & $18(1)$ \\
\hline $\mathrm{C}(5)$ & $-1154(4)$ & $-567(3)$ & $3644(4)$ & $20(1)$ \\
\hline$C(6)$ & $-69(4)$ & $-496(3)$ & 3462(4) & $14(1)$ \\
\hline$C(7)$ & $-233(4)$ & $978(3)$ & 1449(3) & $13(1)$ \\
\hline$C(8)$ & $809(4)$ & $-868(3)$ & $4120(4)$ & $17(1)$ \\
\hline $\mathrm{C}(9)$ & $1387(4)$ & 1974(3) & 2525(4) & $17(1)$ \\
\hline$C(10)$ & 706(4) & $1805(3)$ & 3382(4) & 19(1) \\
\hline$C(11)$ & $990(5)$ & $2762(3)$ & 2104(4) & $25(1)$ \\
\hline$C(12)$ & 2587(4) & 2054(3) & 2916(4) & $22(1)$ \\
\hline$C(13)$ & $1610(4)$ & $1476(3)$ & $436(4)$ & $15(1)$ \\
\hline$C(14)$ & $1602(5)$ & $762(3)$ & $-207(4)$ & $22(1)$ \\
\hline$C(15)$ & $802(4)$ & $2057(3)$ & $-50(4)$ & 19(1) \\
\hline$C(16)$ & 2754(4) & $1822(3)$ & 496(4) & $22(1)$ \\
\hline$C(17)$ & $1835(4)$ & $-2055(3)$ & 3017(4) & $17(1)$ \\
\hline$C(18)$ & 708(4) & $-2097(3)$ & 2477(4) & $21(1)$ \\
\hline$C(19)$ & $1876(5)$ & $-2669(3)$ & 3815(4) & $24(1)$ \\
\hline$C(20)$ & $2670(5)$ & $-2284(3)$ & 2307(4) & $22(1)$ \\
\hline$C(21)$ & $3177(4)$ & $-963(3)$ & 4402(4) & $18(1)$ \\
\hline$C(22)$ & 3393(4) & $-98(3)$ & $4640(4)$ & $20(1)$ \\
\hline$C(23)$ & 4211(4) & $-1306(3)$ & 4046(4) & $24(1)$ \\
\hline$C(24)$ & 2934(4) & $-1361(3)$ & $5345(4)$ & $22(1)$ \\
\hline $\mathrm{N}(1)$ & $2570(3)$ & $-895(2)$ & $664(3)$ & $15(1)$ \\
\hline $\mathrm{O}(1)$ & $1685(3)$ & $-756(2)$ & $1033(2)$ & $15(1)$ \\
\hline $\mathrm{O}(2)$ & $2582(3)$ & $-1289(2)$ & $-65(3)$ & $25(1)$ \\
\hline$C(25)$ & 3551(4) & $-557(3)$ & $1125(4)$ & $17(1)$ \\
\hline$C(26)$ & $3400(4)$ & $-61(3)$ & 1890(4) & $15(1)$ \\
\hline$C(27)$ & 4378(4) & 296(3) & 2249(4) & $21(1)$ \\
\hline$C(28)$ & $5356(5)$ & $135(3)$ & $1898(5)$ & $26(1)$ \\
\hline$C(29)$ & $5450(4)$ & $-409(3)$ & $1173(5)$ & $27(1)$ \\
\hline$C(30)$ & 4531(4) & $-754(3)$ & $755(4)$ & $23(1)$ \\
\hline
\end{tabular}


Table S-6. Selected Bond lengths $[\AA]$ and angles $\left[{ }^{\circ}\right]$ for $\mathbf{1 b}$.

\begin{tabular}{|c|c|c|c|}
\hline $\operatorname{Ir}(1)-C(26)$ & $2.053(5)$ & $C(4)-C(5)$ & $1.369(7)$ \\
\hline $\operatorname{Ir}(1)-C(1)$ & $2.100(5)$ & $\mathrm{C}(4)-\mathrm{H}(4)$ & 0.9500 \\
\hline $\operatorname{Ir}(1)-\mathrm{O}(1)$ & $2.223(3)$ & $C(5)-C(6)$ & $1.391(7)$ \\
\hline $\operatorname{Ir}(1)-P(1)$ & $2.3153(13)$ & $\mathrm{C}(5)-\mathrm{H}(5)$ & 0.9500 \\
\hline $\operatorname{Ir}(1)-P(2)$ & $2.3213(13)$ & $C(6)-C(8)$ & $1.510(7)$ \\
\hline $\operatorname{Ir}(1)-\mathrm{H}(1)$ & $1.594(10)$ & $\mathrm{C}(9)-\mathrm{C}(11)$ & $1.541(7)$ \\
\hline $\mathrm{P}(1)-\mathrm{C}(7)$ & $1.838(5)$ & $\mathrm{C}(9)-\mathrm{C}(12)$ & $1.547(7)$ \\
\hline $\mathrm{P}(1)-\mathrm{C}(13)$ & $1.885(5)$ & $\mathrm{C}(9)-\mathrm{C}(10)$ & $1.548(7)$ \\
\hline $\mathrm{P}(1)-\mathrm{C}(9)$ & $1.886(5)$ & $\mathrm{N}(1)-\mathrm{O}(2)$ & $1.226(5)$ \\
\hline $\mathrm{P}(2)-\mathrm{C}(8)$ & $1.841(5)$ & $\mathrm{N}(1)-\mathrm{O}(1)$ & $1.270(5)$ \\
\hline $\mathrm{P}(2)-\mathrm{C}(21)$ & $1.884(5)$ & $\mathrm{N}(1)-\mathrm{C}(25)$ & $1.452(6$ \\
\hline $\mathrm{P}(2)-\mathrm{C}(17)$ & $1.889(5)$ & $C(25)-C(26)$ & $1.393(7)$ \\
\hline$C(1)-C(2)$ & $1.404(7)$ & $C(25)-C(30)$ & $1.398(7)$ \\
\hline$C(1)-C(6)$ & $1.409(7)$ & $C(26)-C(27)$ & $1.414(7)$ \\
\hline $\mathrm{C}(2)-\mathrm{C}(3)$ & $1.394(7)$ & $\mathrm{C}(27)-\mathrm{C}(28)$ & $1.371(8$ \\
\hline $\mathrm{C}(2)-\mathrm{C}(7)$ & $1.509(7)$ & $\mathrm{C}(28)-\mathrm{C}(29)$ & 1.391( \\
\hline$C(3)-C(4)$ & $1.395(8)$ & $C(29)-C(30)$ & 1.372( \\
\hline $\mathrm{C}(3)-\mathrm{H}(3)$ & 0.9500 & & \\
\hline$C(26)-\operatorname{Ir}(1)-C(1)$ & $174.49(18)$ & $C(26)-C(25)-C(30)$ & $127.4(5)$ \\
\hline $\mathrm{C}(26)-\operatorname{Ir}(1)-\mathrm{O}(1)$ & $76.11(17)$ & $\mathrm{C}(26)-\mathrm{C}(25)-\mathrm{N}(1)$ & $115.3(4)$ \\
\hline $\mathrm{C}(1)-\operatorname{Ir}(1)-\mathrm{O}(1)$ & $98.40(15)$ & $\mathrm{C}(30)-\mathrm{C}(25)-\mathrm{N}(1)$ & $117.3(5)$ \\
\hline $\mathrm{C}(26)-\operatorname{Ir}(1)-\mathrm{P}(1)$ & $101.16(13)$ & $\mathrm{C}(25)-\mathrm{C}(26)-\mathrm{C}(27)$ & $112.0(4)$ \\
\hline $\mathrm{C}(1)-\operatorname{Ir}(1)-\mathrm{P}(1)$ & $79.87(14)$ & $\mathrm{C}(25)-\mathrm{C}(26)-\operatorname{Ir}(1)$ & $116.3(4)$ \\
\hline $\mathrm{O}(1)-\operatorname{Ir}(1)-\mathrm{P}(1)$ & $98.00(9)$ & $C(27)-C(26)-\operatorname{Ir}(1)$ & $131.6(4)$ \\
\hline $\mathrm{C}(26)-\operatorname{Ir}(1)-\mathrm{P}(2)$ & $98.56(13)$ & $\mathrm{C}(28)-\mathrm{C}(27)-\mathrm{C}(26)$ & $122.8(5)$ \\
\hline $\mathrm{C}(1)-\operatorname{Ir}(1)-\mathrm{P}(2)$ & $81.54(14)$ & $\mathrm{C}(28)-\mathrm{C}(27)-\mathrm{H}(27)$ & 118.6 \\
\hline $\mathrm{O}(1)-\operatorname{Ir}(1)-\mathrm{P}(2)$ & $97.16(9)$ & $\mathrm{C}(26)-\mathrm{C}(27)-\mathrm{H}(27)$ & 118.6 \\
\hline $\mathrm{P}(1)-\operatorname{Ir}(1)-\mathrm{P}(2)$ & $157.50(5)$ & $\mathrm{C}(27)-\mathrm{C}(28)-\mathrm{C}(29)$ & $121.6(5)$ \\
\hline $\mathrm{C}(26)-\operatorname{Ir}(1)-\mathrm{H}(1)$ & $102.9(18)$ & $\mathrm{C}(27)-\mathrm{C}(28)-\mathrm{H}(28)$ & 119.2 \\
\hline $\mathrm{C}(1)-\operatorname{Ir}(1)-\mathrm{H}(1)$ & $82.6(18)$ & $\mathrm{C}(29)-\mathrm{C}(28)-\mathrm{H}(28)$ & 119.2 \\
\hline $\mathrm{O}(1)-\operatorname{Ir}(1)-\mathrm{H}(1)$ & $178.7(19)$ & $\mathrm{C}(30)-\mathrm{C}(29)-\mathrm{C}(28)$ & $119.2(5)$ \\
\hline $\mathrm{P}(1)-\operatorname{Ir}(1)-\mathrm{H}(1)$ & $81.3(18)$ & $\mathrm{C}(30)-\mathrm{C}(29)-\mathrm{H}(29)$ & 120.4 \\
\hline $\mathrm{P}(2)-\operatorname{Ir}(1)-\mathrm{H}(1)$ & $83.8(18)$ & $\mathrm{C}(28)-\mathrm{C}(29)-\mathrm{H}(29)$ & 120.4 \\
\hline $\mathrm{O}(2)-\mathrm{N}(1)-\mathrm{O}(1)$ & $120.7(4)$ & $\mathrm{C}(29)-\mathrm{C}(30)-\mathrm{C}(25)$ & $116.9(5)$ \\
\hline $\mathrm{O}(2)-\mathrm{N}(1)-\mathrm{C}(25)$ & $121.7(4)$ & $\mathrm{C}(29)-\mathrm{C}(30)-\mathrm{H}(30)$ & 121.6 \\
\hline $\mathrm{O}(1)-\mathrm{N}(1)-\mathrm{C}(25)$ & $117.5(4)$ & $\mathrm{C}(25)-\mathrm{C}(30)-\mathrm{H}(30)$ & 121.6 \\
\hline $\mathrm{N}(1)-\mathrm{O}(1)-\operatorname{Ir}(1)$ & $114.2(3)$ & & \\
\hline
\end{tabular}


IV. Structural Data for 2a

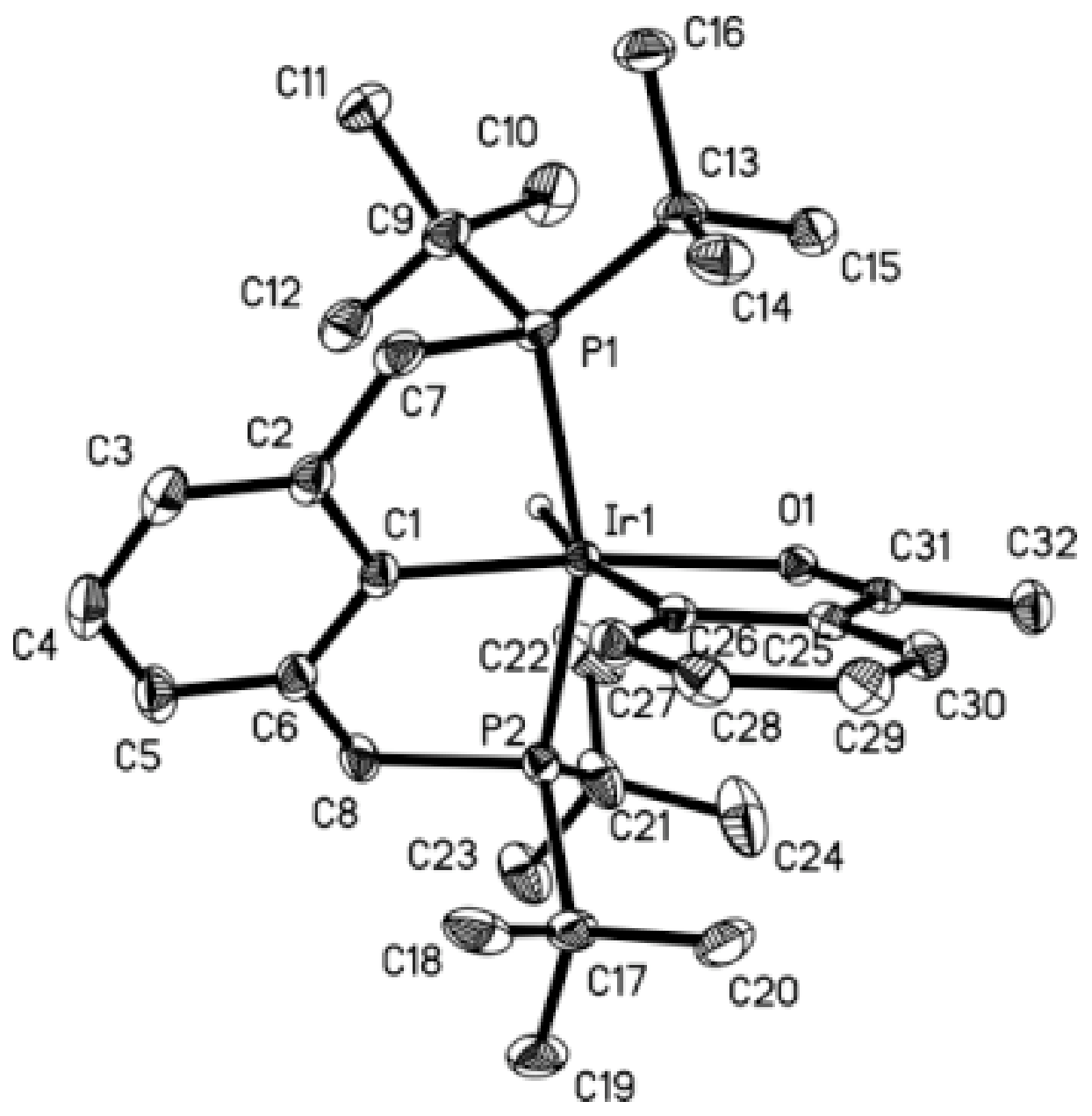

Figure S-3. ORTEP diagram of 2a. Hydrogen atoms omitted for clarity. Thermal ellipsoids are shown at $50 \%$ probability. 
Table S-7. Crystal data and structure refinement for $\mathbf{2 a}$.

\begin{tabular}{|c|c|c|}
\hline Identification code & \multicolumn{2}{|l|}{ irhaoph } \\
\hline Empirical formula & \multicolumn{2}{|l|}{ C32 H51 Ir O P2 } \\
\hline Formula weight & \multicolumn{2}{|l|}{705.87} \\
\hline Temperature & \multicolumn{2}{|l|}{$100(2) \mathrm{K}$} \\
\hline Wavelength & \multicolumn{2}{|l|}{$0.71073 \AA$} \\
\hline Crystal system & \multicolumn{2}{|l|}{ Monoclinic } \\
\hline Space group & \multicolumn{2}{|l|}{$\mathrm{P} 2(1) / \mathrm{c}$} \\
\hline \multirow[t]{3}{*}{ Unit cell dimensions } & $\mathrm{a}=19.4491(9) \AA$ & $\alpha=90^{\circ}$ \\
\hline & $\mathrm{b}=10.4850(5) \AA$ & $\beta=100.886(1)^{\circ}$. \\
\hline & $\mathrm{c}=15.5406(7) \AA$ & $\gamma=90^{\circ}$ \\
\hline Volume & \multicolumn{2}{|l|}{$3112.1(2) \AA^{3}$} \\
\hline Z & \multicolumn{2}{|l|}{4} \\
\hline Density (calculated) & \multicolumn{2}{|l|}{$1.507 \mathrm{Mg} / \mathrm{m}^{3}$} \\
\hline Absorption coefficient & \multicolumn{2}{|l|}{$4.415 \mathrm{~mm}^{-1}$} \\
\hline $\mathrm{F}(000)$ & \multicolumn{2}{|l|}{1432} \\
\hline Crystal size & \multicolumn{2}{|c|}{$0.46 \times 0.18 \times 0.08 \mathrm{~mm}^{3}$} \\
\hline Theta range for data collection & \multicolumn{2}{|l|}{2.13 to $30.52^{\circ}$. } \\
\hline Index ranges & \multicolumn{2}{|c|}{$-27<=\mathrm{h}<=27,-14<=\mathrm{k}<=14,-22<=1<=21$} \\
\hline Reflections collected & \multicolumn{2}{|l|}{37280} \\
\hline Independent reflections & \multicolumn{2}{|c|}{$9490[\mathrm{R}(\mathrm{int})=0.0240]$} \\
\hline Completeness to theta $=30.52^{\circ}$ & \multicolumn{2}{|l|}{$99.9 \%$} \\
\hline Absorption correction & \multicolumn{2}{|c|}{ Semi-empirical from equivalents } \\
\hline Max. and min. transmission & \multicolumn{2}{|l|}{0.7330 and 0.2359} \\
\hline Refinement method & \multicolumn{2}{|c|}{ Full-matrix least-squares on $\mathrm{F}^{2}$} \\
\hline Data / restraints / parameters & \multicolumn{2}{|l|}{$9490 / 1 / 341$} \\
\hline Goodness-of-fit on $\mathrm{F}^{2}$ & \multicolumn{2}{|l|}{1.035} \\
\hline Final $R$ indices $[\mathrm{I}>2 \operatorname{sigma}(\mathrm{I})]$ & \multicolumn{2}{|c|}{$\mathrm{R} 1=0.0198, \mathrm{wR} 2=0.0471$} \\
\hline $\mathrm{R}$ indices (all data) & \multicolumn{2}{|c|}{$\mathrm{R} 1=0.0227, \mathrm{wR} 2=0.0482$} \\
\hline Largest diff. peak and hole & \multicolumn{2}{|c|}{2.074 and -0.658 e. $\AA^{-3}$} \\
\hline
\end{tabular}


Table S-8. Atomic coordinates ( x 10 $0^{4}$ ) and equivalent isotropic displacement parameters $\left(\AA^{2} \times 10^{3}\right)$ for 2a. $\mathrm{U}(\mathrm{eq})$ is defined as one third of the trace of the orthogonalized $\mathrm{U}^{\mathrm{ij}}$ tensor.

\begin{tabular}{|c|c|c|c|c|}
\hline & $\mathrm{x}$ & $y$ & $\mathrm{z}$ & $\mathrm{U}(\mathrm{eq})$ \\
\hline $\operatorname{Ir}(1)$ & 2477(1) & $4117(1)$ & 1792(1) & $13(1)$ \\
\hline $\mathrm{P}(1)$ & $3513(1)$ & $3010(1)$ & 1814(1) & $17(1)$ \\
\hline $\mathrm{P}(2)$ & $1762(1)$ & 5851(1) & 1881(1) & $18(1)$ \\
\hline C(1) & $3106(1)$ & 4999(2) & 2806(1) & $18(1)$ \\
\hline$C(2)$ & $3699(1)$ & $4382(2)$ & $3300(1)$ & $20(1)$ \\
\hline C(3) & $4109(1)$ & $4973(2)$ & 4023(1) & $24(1)$ \\
\hline C(4) & $3947(1)$ & $6192(2)$ & $4273(1)$ & $28(1)$ \\
\hline$C(5)$ & $3387(1)$ & $6837(2)$ & $3775(1)$ & $26(1)$ \\
\hline$C(6)$ & $2975(1)$ & $6260(2)$ & $3044(1)$ & $20(1)$ \\
\hline$C(7)$ & $3883(1)$ & $3082(2)$ & 2994(1) & $22(1)$ \\
\hline$C(8)$ & 2401(1) & $6996(2)$ & 2461(1) & 21(1) \\
\hline $\mathrm{C}(9)$ & $4180(1)$ & $3879(2)$ & $1269(1)$ & $24(1)$ \\
\hline$C(10)$ & $3984(1)$ & $3690(3)$ & $270(1)$ & $34(1)$ \\
\hline$C(11)$ & 4941(1) & $3449(2)$ & $1606(2)$ & $32(1)$ \\
\hline$C(12)$ & $4169(1)$ & $5327(2)$ & $1455(1)$ & $28(1)$ \\
\hline$C(13)$ & $3502(1)$ & $1250(2)$ & 1511(1) & 21(1) \\
\hline$C(14)$ & $3242(1)$ & $462(2)$ & 2224(1) & $28(1)$ \\
\hline$C(15)$ & 2985(1) & $1062(2)$ & $642(1)$ & $26(1)$ \\
\hline$C(16)$ & $4213(1)$ & $684(2)$ & $1420(1)$ & $28(1)$ \\
\hline$C(17)$ & $1083(1)$ & $5751(2)$ & $2603(2)$ & $29(1)$ \\
\hline$C(18)$ & $1488(1)$ & $5466(2)$ & $3528(2)$ & $34(1)$ \\
\hline$C(19)$ & $651(1)$ & $6972(2)$ & $2650(2)$ & $38(1)$ \\
\hline$C(20)$ & $574(1)$ & $4636(2)$ & 2321(2) & $42(1)$ \\
\hline$C(21)$ & $1375(1)$ & $6686(2)$ & $820(1)$ & $31(1)$ \\
\hline$C(22)$ & $1898(2)$ & $6652(2)$ & $200(2)$ & $42(1)$ \\
\hline$C(23)$ & 1201(1) & $8103(2)$ & $942(2)$ & $37(1)$ \\
\hline$C(24)$ & $710(2)$ & $5989(2)$ & $368(2)$ & $50(1)$ \\
\hline $\mathrm{O}(1)$ & $1702(1)$ & $3175(1)$ & $797(1)$ & $16(1)$ \\
\hline$C(25)$ & $1546(1)$ & $1935(2)$ & 1990(1) & $15(1)$ \\
\hline$C(26)$ & 2061(1) & $2725(2)$ & 2506(1) & $15(1)$ \\
\hline$C(27)$ & 2223(1) & $2410(2)$ & $3406(1)$ & 19(1) \\
\hline$C(28)$ & $1903(1)$ & $1415(2)$ & 3759(1) & $22(1)$ \\
\hline$C(29)$ & 1398(1) & $661(2)$ & $3230(1)$ & $23(1)$ \\
\hline $\mathrm{C}(30)$ & $1220(1)$ & $924(2)$ & $2346(1)$ & $20(1)$ \\
\hline$C(31)$ & 1373(1) & $2284(2)$ & $1069(1)$ & $16(1)$ \\
\hline$C(32)$ & $811(1)$ & $1635(2)$ & $423(1)$ & $23(1)$ \\
\hline
\end{tabular}


Table S-9. Selected bond lengths $[\AA]$ and angles $\left[{ }^{\circ}\right]$ for $\mathbf{2 a}$.

\begin{tabular}{|c|c|c|c|}
\hline $\operatorname{Ir}(1)-C(1)$ & $2.0261(17)$ & $C(9)-C(12)$ & $1.547(3)$ \\
\hline $\operatorname{Ir}(1)-C(26)$ & $2.0859(17)$ & $C(13)-C(15)$ & $1.536(3)$ \\
\hline $\operatorname{Ir}(1)-\mathrm{O}(1)$ & $2.1811(12)$ & $C(13)-C(16)$ & $1.536(3)$ \\
\hline $\operatorname{Ir}(1)-P(2)$ & $2.3079(5)$ & $C(13)-C(14)$ & $1.542(3)$ \\
\hline $\operatorname{Ir}(1)-\mathrm{P}(1)$ & $2.3209(5)$ & $\mathrm{C}(17)-\mathrm{C}(18)$ & $1.532(4)$ \\
\hline $\mathrm{P}(1)-\mathrm{C}(7)$ & $1.8401(18)$ & $C(17)-C(19)$ & $1.540(3)$ \\
\hline $\mathrm{P}(1)-\mathrm{C}(13)$ & $1.903(2)$ & $\mathrm{C}(17)-\mathrm{C}(20)$ & $1.541(3)$ \\
\hline $\mathrm{P}(1)-\mathrm{C}(9)$ & $1.909(2)$ & $C(21)-C(22)$ & $1.528(4)$ \\
\hline $\mathrm{P}(2)-\mathrm{C}(8)$ & $1.8366(19)$ & $\mathrm{C}(21)-\mathrm{C}(24)$ & $1.535(3)$ \\
\hline $\mathrm{P}(2)-\mathrm{C}(17)$ & $1.892(2)$ & $C(21)-C(23)$ & $1.544(3)$ \\
\hline $\mathrm{P}(2)-\mathrm{C}(21)$ & $1.893(2)$ & $\mathrm{O}(1)-\mathrm{C}(31)$ & $1.251(2)$ \\
\hline $\mathrm{C}(1)-\mathrm{C}(6)$ & $1.409(3)$ & $C(25)-C(30)$ & $1.402(2)$ \\
\hline $\mathrm{C}(1)-\mathrm{C}(2)$ & $1.416(3)$ & $C(25)-C(26)$ & $1.424(2)$ \\
\hline $\mathrm{C}(2)-\mathrm{C}(3)$ & $1.395(3)$ & $C(25)-C(31)$ & $1.454(2)$ \\
\hline $\mathrm{C}(2)-\mathrm{C}(7)$ & $1.509(3)$ & $\mathrm{C}(26)-\mathrm{C}(27)$ & $1.414(2)$ \\
\hline $\mathrm{C}(3)-\mathrm{C}(4)$ & $1.390(3)$ & $C(27)-C(28)$ & $1.381(3)$ \\
\hline $\mathrm{C}(4)-\mathrm{C}(5)$ & $1.387(3)$ & $\mathrm{C}(28)-\mathrm{C}(29)$ & $1.401(3)$ \\
\hline$C(5)-C(6)$ & $1.398(3)$ & $C(29)-C(30)$ & $1.380(3)$ \\
\hline $\mathrm{C}(6)-\mathrm{C}(8)$ & $1.511(3)$ & $\mathrm{C}(31)-\mathrm{C}(32)$ & $1.500(3)$ \\
\hline $\mathrm{C}(9)-\mathrm{C}(10)$ & $1.540(3)$ & & \\
\hline $\mathrm{C}(9)-\mathrm{C}(11)$ & $1.542(3)$ & & \\
\hline$C(1)-\operatorname{Ir}(1)-C(26)$ & $98.24(7)$ & $\mathrm{C}(1)-\mathrm{C}(6)-\mathrm{C}(8)$ & $118.10(16)$ \\
\hline $\mathrm{C}(1)-\operatorname{Ir}(1)-\mathrm{O}(1)$ & $173.09(6)$ & $\mathrm{C}(2)-\mathrm{C}(7)-\mathrm{P}(1)$ & $106.24(13)$ \\
\hline $\mathrm{C}(26)-\operatorname{Ir}(1)-\mathrm{O}(1)$ & $77.03(6)$ & $\mathrm{C}(6)-\mathrm{C}(8)-\mathrm{P}(2)$ & $108.40(13)$ \\
\hline $\mathrm{C}(1)-\operatorname{Ir}(1)-\mathrm{P}(2)$ & $82.88(6)$ & $\mathrm{C}(15)-\mathrm{C}(13)-\mathrm{C}(16)$ & $108.71(16)$ \\
\hline $\mathrm{C}(26)-\operatorname{Ir}(1)-\mathrm{P}(2)$ & $102.79(5)$ & $\mathrm{C}(15)-\mathrm{C}(13)-\mathrm{C}(14)$ & $108.30(17)$ \\
\hline $\mathrm{O}(1)-\operatorname{Ir}(1)-\mathrm{P}(2)$ & $93.19(3)$ & $C(16)-C(13)-C(14)$ & $106.38(17)$ \\
\hline $\mathrm{C}(1)-\operatorname{Ir}(1)-\mathrm{P}(1)$ & $79.72(6)$ & $\mathrm{C}(15)-\mathrm{C}(13)-\mathrm{P}(1)$ & $108.48(13)$ \\
\hline $\mathrm{C}(26)-\operatorname{Ir}(1)-\mathrm{P}(1)$ & $93.73(5)$ & $\mathrm{C}(16)-\mathrm{C}(13)-\mathrm{P}(1)$ & $115.41(15)$ \\
\hline $\mathrm{O}(1)-\operatorname{Ir}(1)-\mathrm{P}(1)$ & $105.43(3)$ & $\mathrm{C}(14)-\mathrm{C}(13)-\mathrm{P}(1)$ & $109.35(13)$ \\
\hline $\mathrm{P}(2)-\operatorname{Ir}(1)-\mathrm{P}(1)$ & $157.520(17)$ & $\mathrm{C}(22)-\mathrm{C}(21)-\mathrm{C}(24)$ & $107.7(2)$ \\
\hline C(7)-P(1)-C(13) & $105.69(9)$ & $\mathrm{C}(22)-\mathrm{C}(21)-\mathrm{C}(23)$ & $106.8(2)$ \\
\hline$C(7)-P(1)-C(9)$ & 104.60(9) & $C(24)-C(21)-C(23)$ & $109.22(19)$ \\
\hline C(13)-P(1)-C(9) & 109.24(9) & $\mathrm{C}(22)-\mathrm{C}(21)-\mathrm{P}(2)$ & $109.59(15)$ \\
\hline$C(7)-P(1)-\operatorname{Ir}(1)$ & $99.75(6)$ & $\mathrm{C}(24)-\mathrm{C}(21)-\mathrm{P}(2)$ & $109.86(17)$ \\
\hline C(13)-P(1)-Ir(1) & $120.81(6)$ & $\mathrm{C}(23)-\mathrm{C}(21)-\mathrm{P}(2)$ & $113.49(15)$ \\
\hline $\mathrm{C}(9)-\mathrm{P}(1)-\operatorname{Ir}(1)$ & $114.54(7)$ & $\mathrm{C}(31)-\mathrm{O}(1)-\operatorname{Ir}(1)$ & $115.29(11)$ \\
\hline C(8)-P(2)-C(17) & $103.15(9)$ & $C(30)-C(25)-C(26)$ & $122.74(16)$ \\
\hline $\mathrm{C}(8)-\mathrm{P}(2)-\mathrm{C}(21)$ & $104.26(9)$ & $\mathrm{C}(30)-\mathrm{C}(25)-\mathrm{C}(31)$ & $122.67(16)$ \\
\hline $\mathrm{C}(17)-\mathrm{P}(2)-\mathrm{C}(21)$ & $109.57(11)$ & $\mathrm{C}(26)-\mathrm{C}(25)-\mathrm{C}(31)$ & $114.57(15)$ \\
\hline $\mathrm{C}(8)-\mathrm{P}(2)-\operatorname{Ir}(1)$ & $100.69(7)$ & $\mathrm{C}(27)-\mathrm{C}(26)-\mathrm{C}(25)$ & $115.02(15)$ \\
\hline C(17)-P(2)-Ir(1) & $119.18(7)$ & $C(27)-C(26)-\operatorname{Ir}(1)$ & $130.79(13)$ \\
\hline C(21)-P(2)-Ir(1) & $117.25(7)$ & $C(25)-C(26)-\operatorname{Ir}(1)$ & $114.14(12)$ \\
\hline$C(6)-C(1)-C(2)$ & $117.05(17)$ & $\mathrm{C}(28)-\mathrm{C}(27)-\mathrm{C}(26)$ & $122.46(17)$ \\
\hline$C(6)-C(1)-\operatorname{Ir}(1)$ & $121.37(14)$ & $\mathrm{C}(27)-\mathrm{C}(28)-\mathrm{C}(29)$ & $120.81(17)$ \\
\hline C(2)-C(1)-Ir(1) & $121.58(14)$ & $\mathrm{C}(30)-\mathrm{C}(29)-\mathrm{C}(28)$ & $119.21(18)$ \\
\hline $\mathrm{C}(3)-\mathrm{C}(2)-\mathrm{C}(1)$ & $121.16(19)$ & $\mathrm{C}(29)-\mathrm{C}(30)-\mathrm{C}(25)$ & $119.76(18)$ \\
\hline $\mathrm{C}(3)-\mathrm{C}(2)-\mathrm{C}(7)$ & $121.46(18)$ & $\mathrm{O}(1)-\mathrm{C}(31)-\mathrm{C}(25)$ & $118.87(16)$ \\
\hline $\mathrm{C}(1)-\mathrm{C}(2)-\mathrm{C}(7)$ & $117.35(16)$ & $\mathrm{O}(1)-\mathrm{C}(31)-\mathrm{C}(32)$ & $117.79(16)$ \\
\hline$C(5)-C(6)-C(1)$ & $121.10(19)$ & $\mathrm{C}(25)-\mathrm{C}(31)-\mathrm{C}(32)$ & $123.34(16)$ \\
\hline
\end{tabular}




\section{Structural Data for $2 \mathrm{~b}$}

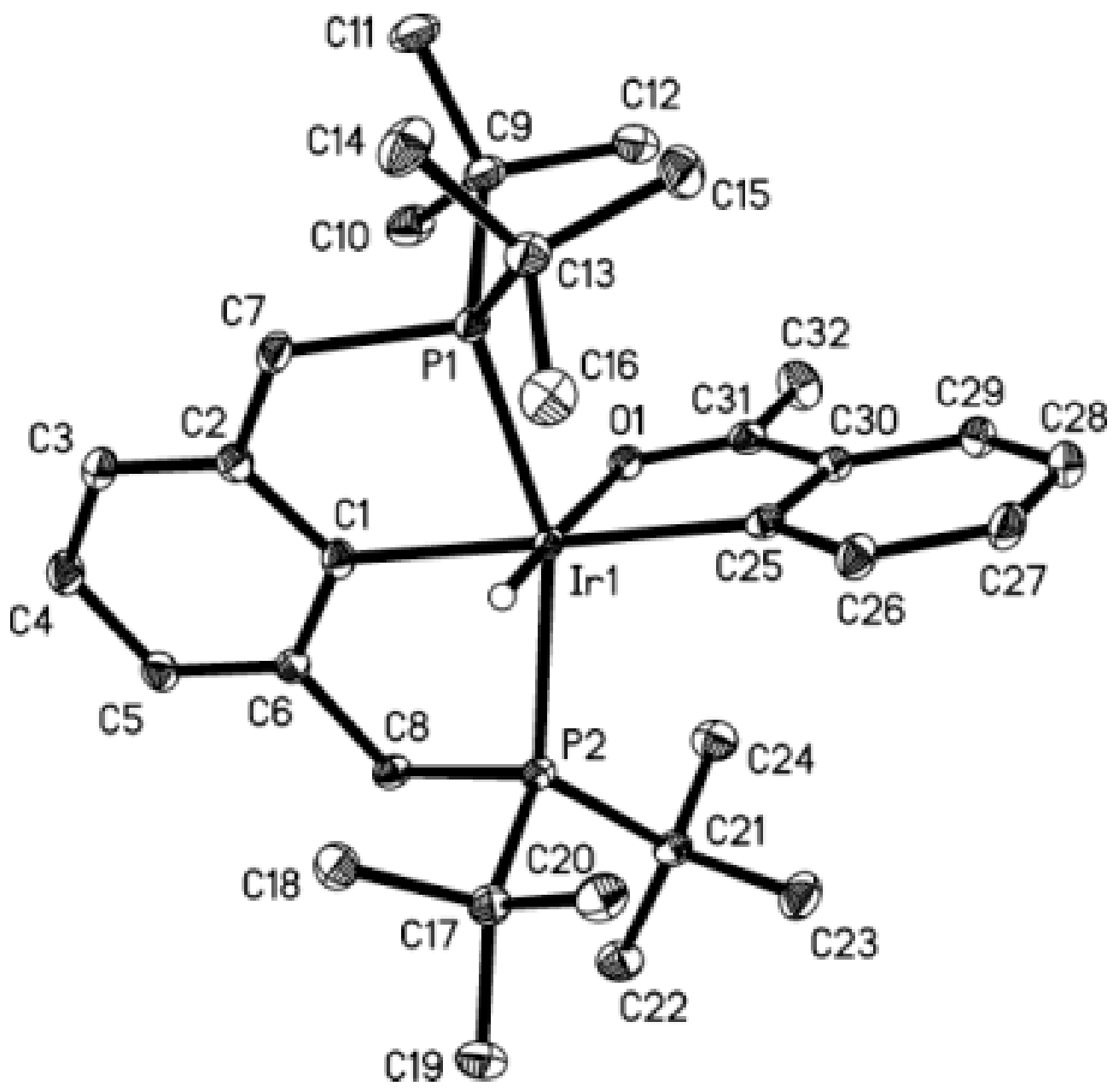

Figure S-4. ORTEP diagram of 2b. Hydrogen atoms omitted for clarity. Thermal ellipsoids are shown at $50 \%$ probability. 
Table S-10. Crystal data and structure refinement for $\mathbf{2 b}$.

\begin{tabular}{|c|c|c|}
\hline Identification code & \multicolumn{2}{|l|}{ irh_cis } \\
\hline Empirical formula & \multicolumn{2}{|l|}{ C32 H51 Ir O P2 } \\
\hline Formula weight & \multicolumn{2}{|l|}{705.87} \\
\hline Temperature & \multicolumn{2}{|l|}{$100(2) \mathrm{K}$} \\
\hline Wavelength & \multicolumn{2}{|l|}{$0.71073 \AA$} \\
\hline Crystal system & \multicolumn{2}{|l|}{ Monoclinic } \\
\hline Space group & \multicolumn{2}{|l|}{$\mathrm{P} 2(1) / \mathrm{c}$} \\
\hline \multirow[t]{3}{*}{ Unit cell dimensions } & $\mathrm{a}=16.0141(8) \AA$ & $\alpha=90^{\circ}$ \\
\hline & $\mathrm{b}=13.3238(6) \AA$ & $\beta=105.463(1)^{\circ}$. \\
\hline & $\mathrm{c}=14.9154(7) \AA$ & $\gamma=90^{\circ}$ \\
\hline Volume & \multicolumn{2}{|l|}{$3067.3(3) \AA^{3}$} \\
\hline $\mathrm{Z}$ & \multicolumn{2}{|l|}{4} \\
\hline Density (calculated) & \multicolumn{2}{|l|}{$1.529 \mathrm{Mg} / \mathrm{m}^{3}$} \\
\hline Absorption coefficient & \multicolumn{2}{|l|}{$4.480 \mathrm{~mm}^{-1}$} \\
\hline $\mathrm{F}(000)$ & \multicolumn{2}{|l|}{1432} \\
\hline Crystal size & \multicolumn{2}{|c|}{$0.31 \times 0.22 \times 0.03 \mathrm{~mm}^{3}$} \\
\hline Theta range for data collection & \multicolumn{2}{|l|}{2.02 to $30.56^{\circ}$} \\
\hline Index ranges & \multicolumn{2}{|c|}{$-22<=\mathrm{h}<=22,-19<=\mathrm{k}<=19,-21<=1<=21$} \\
\hline Reflections collected & \multicolumn{2}{|l|}{37303} \\
\hline Independent reflections & \multicolumn{2}{|c|}{$9367[\mathrm{R}(\mathrm{int})=0.0338]$} \\
\hline Completeness to theta $=30.56^{\circ}$ & \multicolumn{2}{|l|}{$99.7 \%$} \\
\hline Absorption correction & \multicolumn{2}{|c|}{ Semi-empirical from equivalents } \\
\hline Max. and min. transmission & \multicolumn{2}{|l|}{0.8773 and 0.3373} \\
\hline Refinement method & \multicolumn{2}{|c|}{ Full-matrix least-squares on $\mathrm{F}^{2}$} \\
\hline Data / restraints / parameters & \multicolumn{2}{|l|}{$9367 / 0 / 342$} \\
\hline Goodness-of-fit on $\mathrm{F}^{2}$ & \multicolumn{2}{|l|}{1.038} \\
\hline Final $\mathrm{R}$ indices $[\mathrm{I}>2 \operatorname{sigma}(\mathrm{I})]$ & \multicolumn{2}{|c|}{$\mathrm{R} 1=0.0233, \mathrm{wR} 2=0.0564$} \\
\hline $\mathrm{R}$ indices (all data) & \multicolumn{2}{|c|}{$\mathrm{R} 1=0.0274, \mathrm{wR} 2=0.0584$} \\
\hline Largest diff. peak and hole & \multicolumn{2}{|c|}{2.608 and -1.311 e. $\AA^{-3}$} \\
\hline
\end{tabular}


Table S-11. Atomic coordinates ( $\times 10^{4}$ ) and equivalent isotropic displacement parameters $\left(\AA^{2} \times 10^{3}\right)$ for $\mathbf{2 b}$. $U(e q)$ is defined as one third of the trace of the orthogonalized $U^{i j}$ tensor.

\begin{tabular}{|c|c|c|c|c|}
\hline & $\mathrm{x}$ & $y$ & $\mathrm{z}$ & $\mathrm{U}(\mathrm{eq})$ \\
\hline $\mathrm{Ir}(1)$ & $2569(1)$ & 1270(1) & $3490(1)$ & $9(1)$ \\
\hline $\mathrm{P}(1)$ & $2758(1)$ & $-454(1)$ & $3485(1)$ & $12(1)$ \\
\hline $\mathrm{P}(2)$ & $2940(1)$ & 2949(1) & $3705(1)$ & 11(1) \\
\hline $\mathrm{C}(1)$ & $3795(1)$ & $1245(2)$ & $3232(2)$ & $12(1)$ \\
\hline$C(2)$ & $4282(1)$ & $348(2)$ & $3303(1)$ & $14(1)$ \\
\hline$C(3)$ & $5072(1)$ & $312(2)$ & 3074(2) & $16(1)$ \\
\hline $\mathrm{C}(4)$ & $5426(2)$ & 1183(2) & 2814(2) & $17(1)$ \\
\hline $\mathrm{C}(5)$ & 4999(1) & 2092(2) & 2814(1) & $15(1)$ \\
\hline$C(6)$ & 4197(1) & $2123(2)$ & $3017(1)$ & $12(1)$ \\
\hline$C(7)$ & $3942(1)$ & $-564(2)$ & 3691(2) & $16(1)$ \\
\hline $\mathrm{C}(8)$ & $3736(1)$ & $3109(2)$ & $3020(1)$ & $13(1)$ \\
\hline $\mathrm{C}(9)$ & $2320(2)$ & $-1145(2)$ & 2348(2) & $16(1)$ \\
\hline$C(10)$ & $2716(2)$ & $-621(2)$ & $1636(2)$ & $20(1)$ \\
\hline $\mathrm{C}(11)$ & 2572(2) & $-2256(2)$ & $2378(2)$ & $22(1)$ \\
\hline$C(12)$ & $1327(2)$ & $-1064(2)$ & 2011(2) & 19(1) \\
\hline$C(13)$ & $2539(2)$ & $-1223(2)$ & $4465(2)$ & $16(1)$ \\
\hline$C(14)$ & $3113(2)$ & $-2161(2)$ & 4701(2) & $24(1)$ \\
\hline$C(15)$ & $1582(2)$ & $-1526(2)$ & $4239(2)$ & $21(1)$ \\
\hline$C(16)$ & $2732(2)$ & $-560(2)$ & $5335(2)$ & $21(1)$ \\
\hline$C(17)$ & $3563(1)$ & $3240(2)$ & 4944(1) & $16(1)$ \\
\hline$C(18)$ & $4406(1)$ & $2624(2)$ & $5179(2)$ & 19(1) \\
\hline$C(19)$ & $3828(2)$ & $4349(2)$ & 5119(2) & $21(1)$ \\
\hline$C(20)$ & $3026(2)$ & $2925(2)$ & $5609(2)$ & 19(1) \\
\hline $\mathrm{C}(21)$ & 2139(1) & $3999(2)$ & $3245(2)$ & $15(1)$ \\
\hline$C(22)$ & $2577(2)$ & $4986(2)$ & 3082(2) & $20(1)$ \\
\hline $\mathrm{C}(23)$ & $1549(2)$ & $4175(2)$ & $3890(2)$ & $20(1)$ \\
\hline $\mathrm{C}(24)$ & $1565(2)$ & $3660(2)$ & 2302(2) & 19(1) \\
\hline $\mathrm{O}(1)$ & $1745(1)$ & $1380(1)$ & 2030(1) & $13(1)$ \\
\hline$C(25)$ & $1320(1)$ & 1311(1) & $3644(2)$ & $12(1)$ \\
\hline$C(26)$ & $1030(2)$ & $1302(2)$ & $4460(2)$ & $16(1)$ \\
\hline $\mathrm{C}(27)$ & $159(2)$ & $1269(2)$ & $4437(2)$ & 19(1) \\
\hline $\mathrm{C}(28)$ & $-482(2)$ & $1272(2)$ & 3591(2) & 19(1) \\
\hline $\mathrm{C}(29)$ & $-238(1)$ & $1318(2)$ & $2770(2)$ & $16(1)$ \\
\hline $\mathrm{C}(30)$ & $648(1)$ & 1333(1) & 2797(2) & $13(1)$ \\
\hline $\mathrm{C}(31)$ & $950(1)$ & $1376(1)$ & 1958(2) & $13(1)$ \\
\hline$C(32)$ & $337(2)$ & $1417(2)$ & $997(2)$ & $21(1)$ \\
\hline
\end{tabular}


Table S-12. Selected bond lengths $[\AA]$ and angles $\left[^{\circ}\right]$ for $\mathbf{2 b}$.

\begin{tabular}{|c|c|c|c|}
\hline $\operatorname{Ir}(1)-C(25)$ & $2.075(2)$ & $C(9)-C(12)$ & $1.538(3)$ \\
\hline $\operatorname{Ir}(1)-C(1)$ & $2.099(2)$ & $\mathrm{C}(9)-\mathrm{C}(10)$ & $1.542(3)$ \\
\hline $\mathrm{Ir}(1)-\mathrm{O}(1)$ & $2.2307(16)$ & $\mathrm{C}(13)-\mathrm{C}(16)$ & $1.532(3)$ \\
\hline $\operatorname{Ir}(1)-P(2)$ & $2.3158(5)$ & $\mathrm{C}(13)-\mathrm{C}(15)$ & $1.533(3)$ \\
\hline $\operatorname{Ir}(1)-\mathrm{P}(1)$ & $2.3170(5)$ & $C(13)-C(14)$ & $1.536(3)$ \\
\hline $\mathrm{P}(1)-\mathrm{C}(7)$ & $1.844(2)$ & $\mathrm{C}(17)-\mathrm{C}(20)$ & $1.534(3)$ \\
\hline $\mathrm{P}(1)-\mathrm{C}(13)$ & $1.893(2)$ & $\mathrm{C}(17)-\mathrm{C}(18)$ & $1.538(3)$ \\
\hline $\mathrm{P}(1)-\mathrm{C}(9)$ & $1.892(2)$ & $\mathrm{C}(17)-\mathrm{C}(19)$ & $1.540(3)$ \\
\hline $\mathrm{P}(2)-\mathrm{C}(8)$ & $1.846(2)$ & $C(21)-C(24)$ & $1.528(3)$ \\
\hline $\mathrm{P}(2)-\mathrm{C}(17)$ & $1.891(2)$ & $\mathrm{C}(21)-\mathrm{C}(23)$ & $1.535(3)$ \\
\hline $\mathrm{P}(2)-\mathrm{C}(21)$ & $1.899(2)$ & $C(21)-C(22)$ & $1.539(3)$ \\
\hline $\mathrm{C}(1)-\mathrm{C}(6)$ & $1.411(3)$ & $\mathrm{O}(1)-\mathrm{C}(31)$ & $1.249(3)$ \\
\hline$C(1)-C(2)$ & $1.416(3)$ & $C(25)-C(26)$ & $1.414(3)$ \\
\hline $\mathrm{C}(2)-\mathrm{C}(3)$ & $1.397(3)$ & $C(25)-C(30)$ & $1.425(3)$ \\
\hline$C(2)-C(7)$ & $1.508(3)$ & $C(26)-C(27)$ & $1.386(3)$ \\
\hline $\mathrm{C}(3)-\mathrm{C}(4)$ & $1.391(3)$ & $\mathrm{C}(27)-\mathrm{C}(28)$ & $1.399(4)$ \\
\hline$C(4)-C(5)$ & $1.390(3)$ & $C(28)-C(29)$ & $1.383(3)$ \\
\hline$C(5)-C(6)$ & $1.398(3)$ & $\mathrm{C}(29)-\mathrm{C}(30)$ & $1.409(3)$ \\
\hline$C(6)-C(8)$ & $1.508(3)$ & $C(30)-C(31)$ & $1.457(3)$ \\
\hline $\mathrm{C}(9)-\mathrm{C}(11)$ & $1.533(3)$ & $\mathrm{C}(31)-\mathrm{C}(32)$ & $1.507(3)$ \\
\hline$C(25)-\operatorname{Ir}(1)-C(1)$ & 175.91(7) & $C(5)-C(6)-C(1)$ & $121.72(19)$ \\
\hline $\mathrm{C}(25)-\mathrm{Ir}(1)-\mathrm{O}(1)$ & $76.67(7)$ & $\mathrm{C}(5)-\mathrm{C}(6)-\mathrm{C}(8)$ & $120.32(18)$ \\
\hline $\mathrm{C}(1)-\operatorname{Ir}(1)-\mathrm{O}(1)$ & $99.26(7)$ & $\mathrm{C}(1)-\mathrm{C}(6)-\mathrm{C}(8)$ & $117.96(17)$ \\
\hline$C(25)-\operatorname{Ir}(1)-P(2)$ & $100.29(5)$ & $C(2)-C(7)-P(1)$ & $109.36(14)$ \\
\hline $\mathrm{C}(1)-\operatorname{Ir}(1)-\mathrm{P}(2)$ & $79.77(5)$ & $\mathrm{C}(6)-\mathrm{C}(8)-\mathrm{P}(2)$ & $108.14(14)$ \\
\hline $\mathrm{O}(1)-\operatorname{Ir}(1)-\mathrm{P}(2)$ & $97.73(4)$ & $\mathrm{C}(11)-\mathrm{C}(9)-\mathrm{C}(12)$ & $108.60(18)$ \\
\hline$C(25)-\operatorname{Ir}(1)-P(1)$ & $99.04(5)$ & $\mathrm{C}(11)-\mathrm{C}(9)-\mathrm{C}(10)$ & $107.72(18)$ \\
\hline$C(1)-\operatorname{Ir}(1)-\mathrm{P}(1)$ & $81.64(5)$ & $\mathrm{C}(12)-\mathrm{C}(9)-\mathrm{C}(10)$ & $108.85(19)$ \\
\hline $\mathrm{O}(1)-\operatorname{Ir}(1)-\mathrm{P}(1)$ & $96.08(4)$ & $\mathrm{C}(11)-\mathrm{C}(9)-\mathrm{P}(1)$ & $114.30(16)$ \\
\hline $\mathrm{P}(2)-\operatorname{Ir}(1)-\mathrm{P}(1)$ & $158.37(2)$ & $\mathrm{C}(12)-\mathrm{C}(9)-\mathrm{P}(1)$ & $111.25(15)$ \\
\hline $\mathrm{C}(7)-\mathrm{P}(1)-\mathrm{C}(13)$ & $102.84(10)$ & $\mathrm{C}(10)-\mathrm{C}(9)-\mathrm{P}(1)$ & $105.92(15)$ \\
\hline $\mathrm{C}(7)-\mathrm{P}(1)-\mathrm{C}(9)$ & 103.34(10) & $\mathrm{C}(24)-\mathrm{C}(21)-\mathrm{C}(23)$ & 107.61(19) \\
\hline $\mathrm{C}(13)-\mathrm{P}(1)-\mathrm{C}(9)$ & $109.16(10)$ & $\mathrm{C}(24)-\mathrm{C}(21)-\mathrm{C}(22)$ & $107.17(18)$ \\
\hline$C(7)-P(1)-\operatorname{Ir}(1)$ & $101.98(7)$ & $\mathrm{C}(23)-\mathrm{C}(21)-\mathrm{C}(22)$ & $110.56(18)$ \\
\hline $\mathrm{C}(13)-\mathrm{P}(1)-\operatorname{Ir}(1)$ & $118.83(7)$ & $\mathrm{C}(24)-\mathrm{C}(21)-\mathrm{P}(2)$ & $107.28(14)$ \\
\hline $\mathrm{C}(9)-\mathrm{P}(1)-\operatorname{Ir}(1)$ & 117.87(7) & $\mathrm{C}(23)-\mathrm{C}(21)-\mathrm{P}(2)$ & $110.77(15)$ \\
\hline $\mathrm{C}(8)-\mathrm{P}(2)-\mathrm{C}(17)$ & 104.77(10) & $\mathrm{C}(22)-\mathrm{C}(21)-\mathrm{P}(2)$ & $113.17(15)$ \\
\hline $\mathrm{C}(8)-\mathrm{P}(2)-\mathrm{C}(21)$ & 102.38(9) & $\mathrm{C}(31)-\mathrm{O}(1)-\operatorname{Ir}(1)$ & $114.03(13)$ \\
\hline $\mathrm{C}(17)-\mathrm{P}(2)-\mathrm{C}(21)$ & 109.41(10) & $\mathrm{C}(26)-\mathrm{C}(25)-\mathrm{C}(30)$ & $114.9(2)$ \\
\hline$C(8)-P(2)-\operatorname{Ir}(1)$ & 102.97(7) & $C(26)-C(25)-\operatorname{Ir}(1)$ & $130.00(17)$ \\
\hline $\mathrm{C}(17)-\mathrm{P}(2)-\operatorname{Ir}(1)$ & $112.47(7)$ & $C(30)-C(25)-\operatorname{Ir}(1)$ & $115.10(15)$ \\
\hline $\mathrm{C}(21)-\mathrm{P}(2)-\operatorname{Ir}(1)$ & $122.55(7)$ & $\mathrm{C}(27)-\mathrm{C}(26)-\mathrm{C}(25)$ & $122.5(2)$ \\
\hline$C(6)-C(1)-C(2)$ & $116.25(19)$ & $\mathrm{C}(26)-\mathrm{C}(27)-\mathrm{C}(28)$ & $120.9(2)$ \\
\hline$C(6)-C(1)-\operatorname{Ir}(1)$ & $122.25(14)$ & $\mathrm{C}(29)-\mathrm{C}(28)-\mathrm{C}(27)$ & 119.1(2) \\
\hline$C(2)-C(1)-\operatorname{Ir}(1)$ & $121.45(14)$ & $\mathrm{C}(28)-\mathrm{C}(29)-\mathrm{C}(30)$ & $119.7(2)$ \\
\hline $\mathrm{C}(3)-\mathrm{C}(2)-\mathrm{C}(1)$ & 121.73(19) & $\mathrm{C}(29)-\mathrm{C}(30)-\mathrm{C}(25)$ & $122.8(2)$ \\
\hline $\mathrm{C}(3)-\mathrm{C}(2)-\mathrm{C}(7)$ & $120.63(18)$ & $\mathrm{C}(29)-\mathrm{C}(30)-\mathrm{C}(31)$ & $122.5(2)$ \\
\hline $\mathrm{C}(1)-\mathrm{C}(2)-\mathrm{C}(7)$ & $117.55(18)$ & $C(25)-C(30)-C(31)$ & $114.69(19)$ \\
\hline $\mathrm{C}(4)-\mathrm{C}(3)-\mathrm{C}(2)$ & $120.2(2)$ & $\mathrm{O}(1)-\mathrm{C}(31)-\mathrm{C}(30)$ & $119.40(19)$ \\
\hline $\mathrm{C}(5)-\mathrm{C}(4)-\mathrm{C}(3)$ & $119.3(2)$ & $\mathrm{O}(1)-\mathrm{C}(31)-\mathrm{C}(32)$ & $118.13(19)$ \\
\hline$C(4)-C(5)-C(6)$ & $120.38(19)$ & $\mathrm{C}(30)-\mathrm{C}(31)-\mathrm{C}(32)$ & $122.5(2)$ \\
\hline
\end{tabular}

